\title{
A new Perspective on Kinetic Schemes
}

\author{
Michael Junk*
}

\begin{abstract}
Compared to standard numerical methods for hyperbolic systems of conservation laws, Kinetic Schemes model propagation of information by particles instead of waves. In this article, the wave and the particle concept are shown to be closely related. Moreover, a general approach to the construction of Kinetic Schemes for hyperbolic conservation laws is given which summarizes several approaches discussed by other authors. The approach also demonstrates why Kinetic Schemes are particularly well suited for scalar conservation laws and why extensions to general systems are less natural.
\end{abstract}

Keywords. hyperbolic systems of conservation laws, Kinetic Schemes

AMS subject classifications. 35L65, 35L45, 76M25, 82C40

\section{Introduction}

In this article, the connection between general hyperbolic conservation laws and Boltzmann-type transport equations is analyzed which leads to results of both theoretical and numerical interest. The investigations are based on a particular Kinetic Scheme for linear hyperbolic systems. A suitable extension to non-linear systems generalizes several approaches presented by other authors.

Kinetic Schemes have originally been used to construct approximate solutions of gas dynamical Euler equations but the idea has also been extended to other conservation laws. For scalar equations, the approach is very successful because the main ingredient, a suitable equilibrium distribution which generalizes the Maxwellian velocity distribution function of a gas in local thermodynamical equilibrium, is available. Extensions to systems of conservation laws have been proposed in special cases (typically one-dimensional systems and Euler-type equations) but a direct extension of the promising results obtained for the scalar case is difficult which is mainly due to a lack of suitable equilibrium distributions.

*FB Mathematik, Universität Kaiserslautern, 67663 Kaiserslautern, Ger-
many,(junk@mathematik.uni-ki.de).


A main topic of this article is therefore the construction of equilibrium distributions for general hyperbolic systems. After a brief description of the classical case of Euler equations (Section 2), the general framework of kinetic (or particle) formulation is introduced, followed by the definition of a particular Kinetic Scheme in Section 4. In the case of linear hyperbolic systems the kinetic formulation is shown to be closely related to the wave approach based on Fourier analysis. In fact, it turns out in Section 6 that, for a suitable choice of the equilibrium distribution function, the Kinetic Scheme yields the exact solution of the general linear Cauchy problem. In Section 7 , an extension of the approach gives rise to a general construction principle for equilibrium distributions.

A consistency and stability analysis singles out a class of hyperbolic systems for which the kinetic approach seems to be extremely well suited. This class contains all linear equations, all scalar conservation laws and some systems. The members of the class are characterized by the fact that the Kinetic Scheme has infinite order of consistency and that its linearization is the exact solution of the linearized problem. A hyperbolic system belongs to this class if the Jacobian matrix of the fluxes satisfies certain integrability conditions. These conditions are naturally satisfied for scalar equations essentially because any continuous scalar function possesses a primitive due to the fundamental theorem of calculus. A similar argument is not valid for systems due to the fact that a general matrix of continuous functions is not necessarily the gradient of a vector valued function. These considerations give some indication why Kinetic Schemes are well suited for scalar equations and why finding good extensions to systems is more difficult. In Sections 9 to 13, the construction of equilibrium distributions is applied to several specific examples recovering many approaches proposed by other authors.

\section{A particle approach for Euler equations}

To explain how a kinetic (particle) model can be used to approximate solutions of hyperbolic conservation systems, we focus on the important example of Euler equations in gas dynamics (which we write using Einstein's summation convention)

$$
\begin{aligned}
& \partial_{t} \rho+\partial_{x_{j}} \rho u_{j}=0 \\
& \partial_{t} \rho u_{i}+\partial_{x_{j}} \rho u_{i} u_{j}+\partial_{x_{i}} p=0 \\
& \partial_{t} \rho \epsilon+\partial_{x_{j}}(\rho \epsilon+p) u_{j}=0
\end{aligned}
$$

In this continuum description of a gas, the densities of the conserved quantities mass, momentum, and energy are $\rho, \rho \boldsymbol{u}$ and $\rho \epsilon$. The vector $\boldsymbol{u}$ is the velocity of the gas and $p$ is the pressure. For an idcal gas, the pressure satisfies the relationship $p=\rho T$ (the gas constant is suppressed by choosing an appropriate unit for the temperature $T$ ). For simplicity, we consider the case of a mono-atomic gas, where the temperature is related to the energy by $T=2 / 3\left(\epsilon-|\boldsymbol{u}|^{2} / 2\right)$. The idea to solve (1) with a particle 
method has a clear physical origin. Indeed, the continuum description (1) can be refined by taking the atomic structure of the gas into account. For the case of rarefied gases this can be done with the theory of Boltzmann equation. In this approach, the basic quantity is a particle distribution function $f(t, \boldsymbol{x}, \boldsymbol{v})$ which describes the density of gas atoms with velocity $\boldsymbol{v}$ at position $\boldsymbol{x}$ and time $t$. The gas atoms (i.e. the particles) move freely in space unless they undergo collisions. The corresponding evolution of $f$ is given by the Boltzmann equation

$$
\partial_{t} f+v_{j} \partial_{x_{j}} f=Q(f) .
$$

The left hand side of (2) describes free flow of particles whereas collisions are described by the operator $Q$ (for details see [5]). A connection between the two descriptions (1) and (2) is obtained in a limit where particle collisions are dominant. In this asymptotic case, the state variables $f$ and $\rho, \boldsymbol{u}, \epsilon$ as well as the evolutions (1) and (2) are equivalent. More precisely, the particle distribution function $f$ corresponding to the macroscopic densities is an element of the kernel of $Q$ and has the form of a Maxwellian

$$
\mathcal{M}(\rho, \boldsymbol{u}, T ; \boldsymbol{v})=\frac{\rho}{(2 \pi T)^{\frac{3}{2}}} \exp \left(-\frac{|\boldsymbol{v}-\boldsymbol{u}|^{2}}{2 T}\right) .
$$

Since $f=\mathcal{M}$ satisfies $Q(f)=0$, we formally obtain from (2)

$$
\partial_{t} f+v_{j} \partial_{x_{j}} f=0 \quad \text { and } \quad f=\mathcal{M}
$$

To see that this limiting evolution of (2) is equivalent to the Euler system (1), we multiply (4) by $1, \boldsymbol{v}, \frac{1}{2}|\boldsymbol{v}|^{2}$ and integrate over $\boldsymbol{v}$

$$
\partial_{t} \int_{\mathbb{R}^{3}}\left(\begin{array}{c}
1 \\
\boldsymbol{v} \\
\frac{1}{2}|\boldsymbol{v}|^{2}
\end{array}\right) \mathcal{M} d \boldsymbol{v}+\partial_{x_{j}} \int_{\mathbb{R}^{3}} v_{j}\left(\begin{array}{c}
1 \\
\boldsymbol{v} \\
\frac{1}{2}|\boldsymbol{v}|^{2}
\end{array}\right) \mathcal{M} d \boldsymbol{v}=\mathbf{0}
$$

Then, using the explicit form (3) of $\mathcal{M}$, we calculate

$$
\int_{\mathbb{R}^{3}}\left(\begin{array}{c}
1 \\
\boldsymbol{v} \\
\frac{1}{2}|\boldsymbol{v}|^{2}
\end{array}\right) \mathcal{M} d \boldsymbol{v}=\left(\begin{array}{c}
\rho \\
\rho \boldsymbol{u} \\
\rho \epsilon
\end{array}\right), \quad \int_{\mathbb{R}^{3}} v_{j}\left(\begin{array}{c}
1 \\
v_{i} \\
\frac{1}{2}|\boldsymbol{v}|^{2}
\end{array}\right) \mathcal{M} d \boldsymbol{v}=\left(\begin{array}{c}
\rho u_{j} \\
\rho u_{i} u_{j}+p \delta_{i j} \\
(\rho \epsilon+p) u_{j}
\end{array}\right)
$$

so that, indeed, (5) turns into (1).

In the following, we call (4) a kinetic formulation of the Euler equation. Note that the kinetic formulation describes the Euler evolution in terms of a particle ensemble which moves according to the free-flow equation $\partial_{t} f+v_{j} \partial_{x_{j}} f=0$ subject to the constraint $f=\mathcal{M}$ on the velocity distribution of the particles. The big advantage of the kinetic formulation in comparison to the original system (1) is the much simpler structure. The differential operator $\partial_{t}+v_{j} \partial_{x_{j}}$ is scalar and linear in contrast to the nonlinear operator in (1). In particular, any numerical method known for the simple 
advection equation can directly be applied to (4). A corresponding discretization of the Euler system is obtained by multiplying the discretized version of (4) with $1, \boldsymbol{v}$ and $\frac{1}{2}|\boldsymbol{v}|^{2}$ and integrating over $\boldsymbol{v}$. This approach has first been applied to the Euler system in [22], where the Maxwellian distribution has been replaced by another distribution function. In fact, the equivalence of the kinetic formulation to the Euler system just relies on the property (6) of the Maxwellian. Any other function $M(\rho, \boldsymbol{u}, T ; \boldsymbol{v})$ which satisfies (6) also leads to a kinetic formulation if $f=\mathcal{M}$ in (4) is replaced by the constraint $f=M$.

For the classical Maxwellian constraint, Kinetic Schemes have been proposed by several authors. In [20], the kinetic formulation is discretized in both $\boldsymbol{x}$ and $\boldsymbol{v}$. A similar approach is taken in [19] where a more efficient, semi-discrete form of (4) is used. The latter approach was also developed in [6] and extended in several directions by exploiting the possibility to discretize (4) with different methods $[7,8$, 9]. If the constraint function $M$ is nonnegative, the resulting Kinetic Schemes can be set up in such a way that the approximations for $\rho$ and $T$ are also positive (see for example [10]). Moreover, Kinetic Schemes satisfying an entropy inequality are naturally obtained if $M$ is derived using a maximum entropy principle [17].

\section{A particle approach for general hyperbolic systems}

In the following, we will consider general, autonomous hyperbolic problems of the form

$$
\partial_{t} \boldsymbol{U}(t, \boldsymbol{x})+\partial_{x_{j}} \boldsymbol{F}^{j}(\boldsymbol{U}(t, \boldsymbol{x}))=\mathbf{0}, \quad \boldsymbol{U}(0, \boldsymbol{x})=\boldsymbol{U}^{0}(\boldsymbol{x})
$$

with $\boldsymbol{x} \in \mathbb{R}^{d}$. We assume that the unknowns $\boldsymbol{U}=\left(U_{1}, \ldots, U_{m}\right)^{T}$ are contained in a connected open set $\mathcal{S} \subset \mathbb{R}^{m}$ and that $\boldsymbol{F}^{j}: \mathcal{S} \mapsto \mathbb{R}^{m}$ are $C^{1}$-functions. In the generic case $d>1$ and $m>1$, we also assume that $\mathcal{S}$ is simply connected.

Note that (7) is hyperbolic if all linear combinations $\xi_{j} A^{j}(\boldsymbol{U})$ of the Jacobian matrices $A^{j}(\boldsymbol{U})=\nabla \boldsymbol{F}^{j}(\boldsymbol{U})$ of the fluxes have only real eigenvalues for all $\boldsymbol{\xi} \in \mathbb{R}^{d}$ and all $\boldsymbol{U} \in \mathcal{S}$. We speak of a strictly hyperbolic system if $m$ real and distinct eigenvalues exist for $\xi \neq 0$ but our considerations will not be restricted to this case.

In accordance with our considerations above, we call

$$
\left(\partial_{t}+v_{j} \partial_{x_{j}}\right) \Phi=0, \quad \boldsymbol{\Phi}(t, \boldsymbol{x}, \boldsymbol{v})=\boldsymbol{\mu}(\boldsymbol{U}(t, \boldsymbol{x}) ; \boldsymbol{v})
$$

a kinetic formulation of the general system (7) if the constraint function $\boldsymbol{\mu}: \mathcal{S} \times \mathbb{R}^{d} \mapsto$ $\mathbb{R}^{m}$ satisfies the consistency conditions

$$
\langle\boldsymbol{\mu}(\boldsymbol{U} ; \boldsymbol{v}), 1\rangle_{v}=\boldsymbol{U}, \quad \text { and } \quad\left\langle\boldsymbol{\mu}(\boldsymbol{U} ; \boldsymbol{v}), v_{j}\right\rangle_{\boldsymbol{v}}=\boldsymbol{F}^{j}(\boldsymbol{U})
$$


We choose $\langle\cdot, \cdot\rangle_{v}$ to denote integrals over $v \in \mathbb{R}^{d}$ because it later allows us to extend the approach to generalized functions $\mu$ without changing notation.

Again, the kinetic formulation describes the evolution of each $U_{i}$ in terms of a particle ensemble with velocity distribution $\boldsymbol{v} \mapsto \mu_{i}(\boldsymbol{U} ; \boldsymbol{v})$ which moves according to the free flow equation $\left(\partial_{t}+v_{j} \partial_{x_{j}}\right) \Phi_{i}=0$.

The special case of Euler equations is recovered by setting

$$
\boldsymbol{U}=\left(\begin{array}{c}
\rho \\
\rho \boldsymbol{u} \\
\rho \epsilon
\end{array}\right), \quad \boldsymbol{\mu}(\boldsymbol{U} ; \boldsymbol{v})=\left(\begin{array}{c}
1 \\
\boldsymbol{v} \\
\frac{1}{2}|\boldsymbol{v}|^{2}
\end{array}\right) \mathcal{M}(\rho, \boldsymbol{u}, T ; \boldsymbol{v}) .
$$

Here, the first and last component of $\boldsymbol{\mu}$ are the mass and energy distribution of the particle ensemble and the other components represent the momentum distribution.

For the case of scalar equations $(m=1)$ in one space dimension $(d=1)$ a constraint function $\mu$ has been derived in [2, 1]. Although the approach seems to be different from the one in $[11,18]$, where general scalar conservation laws are treated, both approaches turn out to be closely related. In fact, thcy both follow from the construction principle presented below. This construction principle can be viewed as a generalization of the approach in [12] for linear hyperbolic systems in one space dimension which have a complete set of eigenvectors. For the nonlinear system of isentropic Euler equations, the constraint function in [15] is recovered in our general framework at least for the one-dimensional case. Also, the constraint function for the hyperbolic systems in [3] can be obtained from the general construction principle in Section 7.

\section{A Kinetic Scheme}

We have already noted that kinetic formulations can be discretized in many ways giving rise to different Kinetic Schemes. Here, we focus on the construction of constraint functions $\boldsymbol{\mu}$ and therefore restrict ourselves to a simple semi-discrete approximation of (8). The basic idea is to enforce the constraint $\boldsymbol{\Phi}=\boldsymbol{\mu}$ only at $t_{n}=n \Delta t$ which is a purely temporal discretization. Starting with $\mathbf{\Phi}\left(t_{n}, \boldsymbol{x}, \boldsymbol{v}\right)=$ $\boldsymbol{\mu}\left(\boldsymbol{U}^{n}(\boldsymbol{x}) ; \boldsymbol{v}\right)$ we get for $t_{n}<t<t_{n+1}$ as solution of the free flow equation $\left(\partial_{t}+\right.$ $\left.v_{j} \partial_{x_{j}}\right) \Phi=0$

$$
\boldsymbol{\Phi}(t, \boldsymbol{x}, \boldsymbol{v})-\boldsymbol{\mu}\left(\boldsymbol{U}^{n}\left(\boldsymbol{x}-\left(t-t_{n}\right) \boldsymbol{v}\right) ; \boldsymbol{v}\right) .
$$

At the end of the time step, the moment vector $U^{n+1}(\boldsymbol{x})=\left\langle\boldsymbol{\Phi}\left(t_{n \mid 1}, \boldsymbol{x}, \boldsymbol{v}\right), 1\right\rangle_{\boldsymbol{v}}$ is used to re-initialize $\boldsymbol{\Phi}$. Altogether, we can give an inductive definition of the Kinetic Scheme which we are going to consider in the following: 
Let $U^{0}: \mathbb{R}^{d} \mapsto \mathcal{S}$ be the initial value for problem (7) and let $t_{n}=n \Delta t$ for some $\Delta t>0$ and $n \in \mathbb{N}_{0}$. If $U^{n}$ is already constructed and is a function with values in $\mathcal{S}$, we set

$$
\tilde{\boldsymbol{U}}(t, \boldsymbol{x})=\left\langle\boldsymbol{\mu}\left(\boldsymbol{U}^{n}\left(\boldsymbol{x}-\left(t-t_{n}\right) \boldsymbol{v}\right) ; \boldsymbol{v}\right), 1\right\rangle_{\boldsymbol{v}}, \quad t_{n} \leq t \leq t_{n+1}
$$

with the value $\boldsymbol{U}^{n+1}(\boldsymbol{x})=\tilde{\boldsymbol{U}}\left(t_{n+1}, \boldsymbol{x}\right)$ at the end of the time step.

To check that $\tilde{U}$ is an approximation of the solution of (7) we use a Taylor expansion around $t_{0}=0$ which suffices due to the iterative structure of the algorithm. With (9) we have

$$
\tilde{\boldsymbol{U}}(0, \boldsymbol{x})=\left\langle\boldsymbol{\mu}\left(\boldsymbol{U}^{0}(\boldsymbol{x}) ; \boldsymbol{v}\right), 1\right\rangle_{\boldsymbol{v}}=\boldsymbol{U}^{0}(\boldsymbol{x})
$$

and

$$
\left.\partial_{t} \tilde{\boldsymbol{U}}(t, \boldsymbol{x})\right|_{t=0}=\left\langle-v_{j} \partial_{x_{j}} \boldsymbol{\mu}\left(\boldsymbol{U}^{0}(\boldsymbol{x}) ; \boldsymbol{v}\right), 1\right\rangle_{\boldsymbol{v}}=-\partial_{x_{j}} \boldsymbol{F}^{j}\left(\boldsymbol{U}^{0}(\boldsymbol{x})\right)
$$

so that indeed

$$
\tilde{\boldsymbol{U}}(t, \boldsymbol{x})=\boldsymbol{U}^{0}(\boldsymbol{x})-\partial_{x_{j}} \boldsymbol{F}^{j}\left(\boldsymbol{U}^{0}(\boldsymbol{x})\right) \Delta t+\mathcal{O}\left(\Delta t^{2}\right)
$$

In other words, the consistency conditions (9) guarantee that $\tilde{U}$ is at least a first order consistent approximation of the solution of (7).

In general, the consistency conditions do not determine $\boldsymbol{\mu}$ uniquely and there should be a selection mechanism to single out an appropriate constraint function. One possibility is to select a constraint function which is optimal with respect to a convex functional (entropy), while satisfying the consistency conditions. As a by-product, the resulting Kinetic Scheme also satisfies an entropy inequality (see [17]). In this work, we pursue a different optimality condition: we select the constraint function $\mu$ which maximizes the order of consistency of the semi-discrete Kinetic Scheme introduced above. We will see that the maximal order of consistency obtainable with Kinetic Schemes of the above type depends on structural properties of the hyperbolic system. For somc systcms (including all linear and nonlinear scalar ones) the maximal order of consistency turns out to be infinite. For general systems, however, there is an upper bound $n_{0} \geq 1$ for the maximal consistency order of the Kinetic Schemes proposed above. We remark that other discretizations of (8) can, of course, lead to Kinetic Schemes with higher orders of consistency. The semidiscrete approximation chosen here, however, is very well suited for the construction of constraint functions which is our main objective.

\section{The wave approach}

To illustrate the similarities between the particle approach bascd on a kinctic formulation and the more common wave approach, let us briefly recollect the case of 
a linear hyperbolic system where the flux functions are of the form $\boldsymbol{F}^{j}(\boldsymbol{U})=A^{j} \boldsymbol{U}$ with constant matrices $A^{j} \in \mathbb{R}^{m \times m}$

$$
\partial_{t} \boldsymbol{U}(t, \boldsymbol{x})+A^{j} \partial_{x_{j}} \boldsymbol{U}(t, \boldsymbol{x})=\mathbf{0}, \quad \boldsymbol{U}(0, \boldsymbol{x})=\boldsymbol{U}^{0}(\boldsymbol{x})
$$

Applying the Fourier transform in the space variable, we obtain a system of ordinary differential equations

$$
\partial_{t} \hat{\boldsymbol{U}}(t, \boldsymbol{\xi})+i \xi_{j} A^{j} \hat{\boldsymbol{U}}(t, \boldsymbol{\xi})=\mathbf{0}, \quad \hat{\boldsymbol{U}}(0, \boldsymbol{\xi})=\hat{\boldsymbol{U}}^{0}(\boldsymbol{\xi})
$$

(with $i$ being the imaginary unit) which has the solution

$$
\hat{\boldsymbol{U}}(t, \boldsymbol{\xi})=\hat{E}_{t}(\boldsymbol{\xi}) \hat{\boldsymbol{U}}^{0}(\boldsymbol{\xi}), \quad \hat{E}_{t}(\boldsymbol{\xi})=\exp \left(-i t \xi_{j} A^{j}\right)
$$

Transforming back, we can write

$$
\left.\boldsymbol{U}(t, \boldsymbol{x})=\frac{1}{(2 \pi)^{d}} \int_{\mathbb{R}^{d}} \exp \left(i(\boldsymbol{\xi} \cdot \boldsymbol{x}) \mathrm{I}-i t \xi_{j} A^{j}\right)\right) \hat{\boldsymbol{U}}^{0}(\boldsymbol{\xi}) d \boldsymbol{\xi}
$$

which shows that the solution of (11) is a superposition of plane waves

$$
\left.\boldsymbol{W}_{\boldsymbol{\xi}}(t, \boldsymbol{x})=\exp \left(i(\boldsymbol{\xi} \cdot \boldsymbol{x}) \mathrm{I}-i t \xi_{j} A^{j}\right)\right) \hat{\boldsymbol{U}}^{0}(\boldsymbol{\xi})
$$

Note that in the strictly hyperbolic case, $\boldsymbol{W}_{\boldsymbol{\xi}}$ can be written as

$$
\boldsymbol{W}_{\boldsymbol{\xi}}(t, \boldsymbol{x})=V_{\boldsymbol{\xi}} \operatorname{diag}\left(\exp \left(i \boldsymbol{\xi} \cdot \boldsymbol{x}-i t|\boldsymbol{\xi}| \lambda_{\boldsymbol{\xi}}^{k}\right)\right) V_{\boldsymbol{\xi}}^{-1} \hat{\boldsymbol{U}}^{0}(\boldsymbol{\xi})
$$

where $\lambda_{\boldsymbol{\xi}}^{k}$ are the eigenvalues of $\xi_{j} A^{j} /|\boldsymbol{\xi}|$ and $V_{\boldsymbol{\xi}}$ is a matrix containing the corresponding eigenvectors as columns. Hence, each component of the vector $\boldsymbol{W}_{\boldsymbol{\xi}}$ is a linear combination of the scalar plane waves $\exp \left(i \boldsymbol{\xi} \cdot \boldsymbol{x}-i t|\boldsymbol{\xi}| \lambda_{\boldsymbol{\xi}}^{k}\right)$ which travel in direction $\boldsymbol{\xi} /|\boldsymbol{\xi}|$ with wave speed $\lambda_{\boldsymbol{\xi}}^{k}$ having the wave length $2 \pi /|\boldsymbol{\xi}|(\boldsymbol{\xi}$ is called wave vector). For non-strictly hyperbolic systems, a similar consideration is possible based on a Jordan representation of $\xi_{j} A^{j}$.

\section{Equivalence of wave and particle approach}

In the previous section, we have seen that the solution of a linear hyperbolic system can be given in terms of a superposition of plane waves. Using the notation of eq. (12) and $\mathcal{F}$ to denote the Fourier transform we have

$$
\boldsymbol{U}(t, \boldsymbol{x})=\left.\mathcal{F}_{\boldsymbol{\xi}}^{-1}\left(\hat{E}_{t}(\boldsymbol{\xi}) \hat{\boldsymbol{U}}^{0}(\boldsymbol{\xi})\right)\right|_{\boldsymbol{x}}
$$


The reformulation of the wave approach into a particle formulation simply relies on the property of the Fourier transform to convert products into convolutions. Denoting $E_{t}:=\mathcal{F}^{-1} \hat{E}_{t}$ we obtain formally $\boldsymbol{U}(t, \boldsymbol{x})=E_{t} * \boldsymbol{U}^{0}(\boldsymbol{x})$, or more explicitly

$$
\boldsymbol{U}(t, \boldsymbol{x})=\left\langle E_{t}(\boldsymbol{y}) \boldsymbol{U}^{0}(\boldsymbol{x}-\boldsymbol{y}), 1\right\rangle_{\boldsymbol{y}} .
$$

(To avoid technicalities at this point we proceed purely formal. Actually, $E_{t}=$ $\mathcal{F}_{\xi}^{-1} \exp \left(-i t \xi_{j} A^{j}\right)$ has to be interpreted in the sense of distributions which we do later.) Using the fact that

$$
\left.\mathcal{F}_{\boldsymbol{\xi}}^{-1} \phi(t \boldsymbol{\xi})\right|_{t \boldsymbol{v}}=\frac{1}{(2 \pi)^{d}} \int_{\mathbb{R}^{d}} \phi(t \boldsymbol{\xi}) \exp (i \boldsymbol{\xi} \cdot t \boldsymbol{v}) d \boldsymbol{\xi}=\frac{1}{t^{d}} \frac{1}{(2 \pi)^{d}} \int_{\mathbb{R}^{d}} \phi(\boldsymbol{\eta}) \exp (i \boldsymbol{\eta} \cdot \boldsymbol{v}) d \boldsymbol{\eta}
$$

for any test function $\phi$, we find accordingly $E_{t}(v t)=E_{1}(v) / t^{d}$. Hence, the change of variables $\boldsymbol{y}=\boldsymbol{t} \boldsymbol{v}$ in (13) yields

$$
\boldsymbol{U}(t, \boldsymbol{x})=\left\langle E_{1}(\boldsymbol{v}) \boldsymbol{U}^{0}(\boldsymbol{x}-t \boldsymbol{v}), 1\right\rangle_{\boldsymbol{v}} .
$$

'l'his result suggests to introduce the vector constraint function

$$
\stackrel{\circ}{\boldsymbol{\mu}}_{l i n}(\boldsymbol{U} ; \boldsymbol{v}):=E(\boldsymbol{v}) \boldsymbol{U}, \quad E=E_{1}=\mathcal{F}_{\boldsymbol{\xi}}^{-1} \exp \left(-i \xi_{j} A^{j}\right) .
$$

Then, the solution (14) of the linear hyperbolic system coincides with the approximation $\tilde{U}$ obtained with the Kinetic Scheme defined in Section 4

$$
\tilde{U}(t, x)=\left\langle\stackrel{\circ}{\text { lin }}_{\text {in }}\left(U^{0}(x-t v) ; v\right), 1\right\rangle_{v} .
$$

This remarkable result is partly related to our choice of the Kinetic Scheme but it demonstrates that the concept of kinetic formulations is intimately related to hyperbolic equations. In particular, the derivation shows that solving the free transport equation together with velocity averaging is closely connected to convolution.

Since the Kinetic Scheme based on $\stackrel{\circ}{\boldsymbol{\mu}}_{\text {lin }}$ yields the exact solution, it is evident, that the consistency conditions (9) are satisfied. This property can also be checked directly. Translating $\boldsymbol{v}$-moments of $E$ into $\boldsymbol{\xi}$-derivatives of the Fourier transform at $\xi=\mathbf{0}$, we have

$$
\langle E, 1\rangle_{v}=\mathcal{F}(E)(\mathbf{0})=\left.\exp \left(-i \xi_{j} A^{j}\right)\right|_{\xi=0}=\mathrm{I}
$$

and

$$
\left\langle E, v_{k}\right\rangle_{\boldsymbol{v}}=\mathcal{F}\left(v_{k} E\right)(\mathbf{0})=\left.i \partial_{\xi_{k}} \exp \left(-i \xi_{j} A^{j}\right)\right|_{\boldsymbol{\xi}=0}=A^{k}
$$

so that

$$
\left\langle\stackrel{\circ}{\boldsymbol{\mu}}_{l i n}(\boldsymbol{U}), 1\right\rangle_{\boldsymbol{v}}=\langle E, 1\rangle_{\boldsymbol{v}} \boldsymbol{U},=\boldsymbol{U} \quad\left\langle\stackrel{\circ}{\boldsymbol{\mu}}_{\text {lin }}(\boldsymbol{U}), v_{k}\right\rangle_{\boldsymbol{v}}=\left\langle E, v_{k}\right\rangle_{\boldsymbol{v}} \boldsymbol{U}=A^{k} \boldsymbol{U} .
$$




\section{General constraint functions}

For general linear hyperbolic problems like (11) we have found an optimal constraint function

$$
\stackrel{\circ}{\boldsymbol{\mu}}_{\text {lin }}(\boldsymbol{U} ; \boldsymbol{v}):=E(\boldsymbol{v}) \boldsymbol{U}, \quad E=\mathcal{F}_{\boldsymbol{\xi}}^{-1} \exp \left(-i \xi_{j} A^{j}\right)
$$

for which the corresponding Kinetic Scheme even yields the exact solution. In [12], the same constraint has been derived for the linear case in a single space dimension ( $d=1)$ if $A^{1}$ has a complete set of eigenvectors. The authors are able to extend the construction of $\boldsymbol{\mu}$ to nonlinear systems if the flux can be put in the form

$$
\boldsymbol{F}^{1}(\boldsymbol{U})=A^{1}(\boldsymbol{U}) \boldsymbol{U}
$$

with $A^{1}(U)$ having point-wise the same properties as required for the linear case (such a representation is possible if the system admits a convex entropy). With this extension, however, the optimality in the linear case is lost (as far as recovering exact solutions is concerned). We therefore propose a different generalization of (18). First, we rewrite (18) as integral over the matrix $E$ which is constant with respect to $U$,

$$
{\stackrel{\circ}{\mu_{l i n}}}_{\text {lU }}(U)=\int_{0}^{U} E:=\int_{0}^{1} E \dot{\gamma}(s) d s
$$

where $\gamma:[0,1] \mapsto \mathbb{R}^{m}$ is a curve in the state space $\mathcal{S}=\mathbb{R}^{m}$ which connects the origin with $U$. To obtain an expression similar to (19) in the case of general systems, we assume in the following that $\mathbf{0} \in \mathcal{S}$ and $\boldsymbol{F}^{j}(\mathbf{0})=\mathbf{0}$ for $j=1, \ldots, d$. This can always be achieved by selecting some point $\tilde{U} \in \mathcal{S}$ and going over to the fluxes $\tilde{\boldsymbol{F}}^{j}(\boldsymbol{V})=\boldsymbol{F}^{j}(\boldsymbol{V}+\tilde{\boldsymbol{U}})-\boldsymbol{F}^{j}(\tilde{\boldsymbol{U}})$ defined on $\tilde{\mathcal{S}}=\mathcal{S}-\tilde{\boldsymbol{U}}$ which certainly contains 0.

A straight forward generalization of (18) and (19) to the nonlinear case is obtained if we replace the constant matrices $A^{j}$ by the flux derivatives $A^{j}(\boldsymbol{U})=\nabla \boldsymbol{F}^{j}(\boldsymbol{U})$. Obviously, the matrix $E$ then depends on $U \in \mathcal{S} \subset \mathbb{R}^{m}$

$$
E(\boldsymbol{U})=\mathcal{F}_{\boldsymbol{\xi}}^{-1} \exp \left(-i \xi_{j} A^{j}(\boldsymbol{U})\right)
$$

so that the line integral of $E$ in (19) is no longer trivial. Since we cannot expect that the line integrals are independent of the chosen curves in the state spacc $\mathcal{S}$, wc have to fix properties of the parameterization. If $\boldsymbol{\gamma}(\boldsymbol{U} ; s)$ is the parameterization of a curve connecting the origin with $U$ in $\mathcal{S}$, we require that the $U$-dependence of $\gamma$ is reasonably nice and that the integrals of $E$ along the curves are well defined (we speak of $\boldsymbol{F}$-adnissible curves - for details see Definition 5 in the appendix). If $\Gamma_{\boldsymbol{U}}$ is the graph of the parameterization $s \mapsto \gamma(U ; s)$, we define the line integral of the matrix $E$ as

$$
\int_{\Gamma_{U}} E:=\int_{0}^{1} E(\boldsymbol{\gamma}(\boldsymbol{U} ; s)) \dot{\boldsymbol{\gamma}}(\boldsymbol{U} ; s) d s
$$


where $\dot{\gamma}$ refers to the $s$-derivative of $\boldsymbol{\gamma}$. Finally, the proposed generalization of (19) to the case of nonlinear hyperbolic systems is given by

$$
\stackrel{\circ}{\mu}(\boldsymbol{U}):=\int_{\Gamma_{U}} E, \quad E(\boldsymbol{U})=\mathcal{F}_{\boldsymbol{\xi}}^{-1} \exp \left(-i \xi_{j} A^{j}(\boldsymbol{U})\right)
$$

A rigorous description of the mathematical properties of $\dot{\mu}$ is given in the appendix (where the approach is even generalized to entropy conservation laws related to the hyperbolic system). It turns out that each component of $U \mapsto \stackrel{\mu}{U}(U)$ is contained in $\mathscr{K}$, the set of continuous mappings from $\mathcal{S}$ into the space $\mathscr{E}^{\prime}\left(\mathbb{R}^{d}\right)$ of compactly supported distributions which satisfy a locally uniform estimate (see Definition 6 for details). As in the case of linear systems, one can show that $\stackrel{\circ}{\mu}$ satisfies the consistency conditions (9). Using the fact that $\langle E, 1\rangle=\mathrm{I}$ and $\left\langle E, v_{k}\right\rangle=A^{k}(\boldsymbol{U})$ for every $U \in \mathcal{S}$ (see (16) and (17)), we get

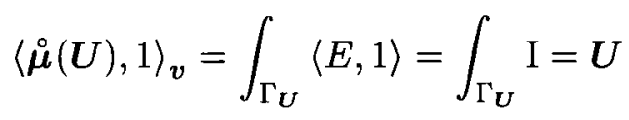

and

$$
\left\langle\stackrel{\circ}{\boldsymbol{\mu}}(\boldsymbol{U}), v_{k}\right\rangle_{\boldsymbol{v}}=\int_{\Gamma_{\boldsymbol{U}}}\left\langle E, v_{k}\right\rangle=\int_{\Gamma_{\boldsymbol{U}}} A^{k}=\int_{\Gamma_{\boldsymbol{U}}} \nabla \boldsymbol{F}^{k}=\boldsymbol{F}^{k}(\boldsymbol{U}) .
$$

We conclude that, for any (autonomous) hyperbolic system the constraint function $\stackrel{\mu}{\mu}$ satisfies the consistency conditions required for a kinetic formulation. In fact, one can show that the Kinetic Scheme based on $\stackrel{\mu}{\mu}$ maximizes the order of consistency. Morc prcciscly, for $\mu \in \mathscr{K}^{m}$, the Kinetic Scheme defined in Section 4 yields the approximation

$$
\tilde{\boldsymbol{U}}(t, \boldsymbol{x}):=\left\langle\boldsymbol{\mu}\left(\boldsymbol{U}^{0}(\boldsymbol{x}-\boldsymbol{v} t) ; \boldsymbol{v}\right), 1\right\rangle_{\boldsymbol{v}}
$$

where $\boldsymbol{U}^{0}$ is taken from the set of initial values

$$
\mathscr{J}:=\left\{\boldsymbol{V}: \mathbb{R}^{d} \mapsto \mathcal{S}: \boldsymbol{V} \in C^{\infty}\left(\mathbb{R}^{d}\right)^{m}, \boldsymbol{V}\left(\mathbb{R}^{d}\right) \subset \subset \mathcal{S}\right\} .
$$

If $\boldsymbol{U}(t, \boldsymbol{x})$ is the corresponding classical solution of $(7)$ on $(-T, T) \times \mathbb{R}^{d}$ for some $T>0$, we define the consistency as

$$
\operatorname{con}(\boldsymbol{\mu}):=\inf \left\{n-1: n \in \mathbb{N}_{0},\left.\partial_{t}^{n} \boldsymbol{U}\right|_{t=0} \neq\left.\partial_{t}^{n} \tilde{\boldsymbol{U}}\right|_{t=0} \text { for some } \boldsymbol{U}^{0} \in \mathscr{J}\right\} .
$$

With this notation, the optimality of $\dot{\mu}$ can be formulated as $\operatorname{con}(\stackrel{\circ}{\mu}) \geq \operatorname{con}(\boldsymbol{\mu})$ for all $\mu \in \mathscr{K}^{m}$. The following theorem relates the maximal order of consistency $\operatorname{con}(\stackrel{\mu}{\mu})$ to properties of the conservation law (7). Note that a matrix valued function $B: \mathcal{S} \mapsto \mathbb{R}^{n \times d}$ is called exact if $B=\nabla \boldsymbol{b}$ for some $\boldsymbol{b}: \mathcal{S} \mapsto \mathbb{R}^{n}$.

Theorem 1 An explicit expression for $\operatorname{con}(\stackrel{\circ}{\mu})$ is given by

$$
\operatorname{con}(\stackrel{\boldsymbol{\mu}}{)})=\sup \left\{n \in \mathbb{N}:\left(\xi_{j} A^{j}\right)^{\bar{n}-1} A^{k} \text { is exact } \forall \bar{n} \leq n, \boldsymbol{\xi} \in \mathbb{R}^{d}, k=1, \ldots, d\right\} \text {. }
$$


For systems in one space dimension $(d=1)$, the condition reduces to exactness of $\bar{n}$-fold products of the Jacobian $A^{1}$ for all $\bar{n} \leq n$. In the scalar case $(m=1)$ and for linear systems, the maximal consistency order is always infinite. Since $\operatorname{con}(\stackrel{\circ}{\mu}) \geq 1$ for any system, the Kinetic Scheme based on $\dot{\mu}$ is always consistent. If $\operatorname{con}(\boldsymbol{\mu})=\infty$ for some $\boldsymbol{\mu} \in \mathscr{K}^{m}$, then $\boldsymbol{\mu}$ is essentially given by $\stackrel{\circ}{\boldsymbol{\mu}}$, i.e. $\boldsymbol{\mu}=\stackrel{\circ}{\boldsymbol{\mu}}_{\eta}+\boldsymbol{C}$ where $\boldsymbol{C} \in\left[\mathscr{E}^{\prime}\left(\mathbb{R}^{d}\right)\right]^{m}$ is independent of $\boldsymbol{U}$ and satisfies $\langle\boldsymbol{C}, 1\rangle=\mathbf{0}$.

Proofs for the results in this theorem can be found in [13] and a forthcoming article [14]. We remark that the infinite order of consistency in the case of scalar equations follows from the fact that $\left(\xi_{j} A^{j}\right)^{\bar{n}-1} A^{k}$ is a continuous, scalar function in the scalar state variable $U$. Using the fundamental theorem of calculus, a primitive can be obtained simply by integrating with variable upper bound which shows exactness for any $\xi, \bar{n}$ and $k$. Already for systems in one space dimension, the situation is very much different. Even $\left(A^{1}\right)^{2}=\nabla \boldsymbol{F}^{1} \nabla \boldsymbol{F}^{1}$ need not be exact so that the Kinetic Scheme will, in general, yield only a first order consistent method. Nevertheless, there exist systems for which the exactness conditions are satisfied. Some examples will be mentioned in Sections 11 and 12 .

\section{Some remarks on stability}

Up to now, we have only investigated consistency properties of the Kinetic Scheme. However, consistency alone does not fully describe the behavior of the scheme. The second important concept besides consistency is stability. Since a theory of stability for general hyperbolic systems of conservation laws is not available, we resort to some heuristic arguments. First, we mention the idea of linear stability and then consider the approach of modified equations for the case of one-dimensional systems.

\subsection{Linear stability}

In general, the constraint function $\boldsymbol{U} \mapsto \stackrel{\circ}{\boldsymbol{\mu}}(\boldsymbol{U})$ depends nonlinearly on $\boldsymbol{U}$ so that the Kinetic Scheme based on $\stackrel{\circ}{\mu}$ is also nonlinear. However, if the initial value varies only slightly around some value $\bar{U} \in \mathcal{S}$, one can linearize the Kinetic Scheme and study the stability properties of the resulting scheme which approximates the linearization of (7)

$$
\partial_{t} \boldsymbol{W}+A^{j}(\overline{\boldsymbol{U}}) \partial_{x_{j}} \boldsymbol{W}=\mathbf{0},\left.\quad \boldsymbol{W}\right|_{t=0}=\boldsymbol{W}^{0}=\boldsymbol{U}^{0}-\overline{\boldsymbol{U}} .
$$

A corresponding linearization of the Kinetic Scheme relies on

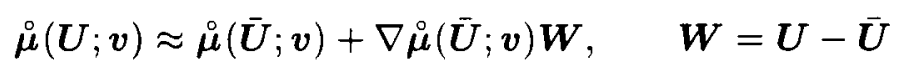


where the gradient of $\dot{\mu}$ is taken with respect to the state variable $U$. To calculate the derivative of $\dot{\mu}$ into some direction $\boldsymbol{e} \in \mathbb{R}^{m}$, we select a curve $\delta \in C^{1}\left([0,1], \mathbb{R}^{m}\right)$ which satisfies $\delta(0)=0$ and $\dot{\delta}(0)=e$. Then

$$
\nabla \stackrel{\circ}{\boldsymbol{\mu}}(\boldsymbol{U}) \boldsymbol{e}=\lim _{h \rightarrow 0} \frac{1}{h}\left(\int_{\Gamma_{U+\delta(h)}} E-\int_{\Gamma_{\boldsymbol{U}}} E\right) .
$$

Introducing the graph $\Delta_{h}(\boldsymbol{U}, \boldsymbol{e})=\boldsymbol{U}+\delta([0, h])$, we set up the oriented closed curve $C_{h}(\boldsymbol{U}, \boldsymbol{e}):=\Gamma_{\boldsymbol{U}+\boldsymbol{\delta}(h)}-\Delta_{h}(\boldsymbol{U}, \boldsymbol{e})-\Gamma_{\boldsymbol{U}}$ (see Figure 1).

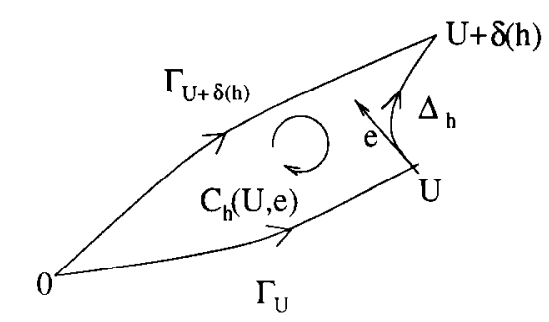

Figure 1: Closed curve in state space

Hence, (22) transforms into

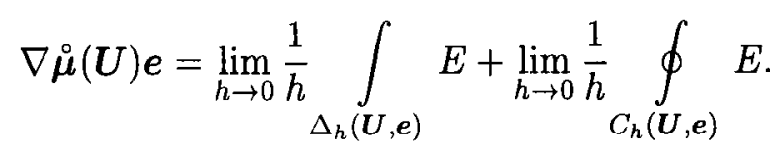

Using the mean value theorem, the first integral reduces to $E(U) e$ and the second one can be rewritten as

$$
q(\boldsymbol{U}) \boldsymbol{e}=\left.\frac{d}{d h} \oint_{C_{h}(\boldsymbol{U}, \boldsymbol{e})} E\right|_{h=0}
$$

Altogether, we find $\nabla \stackrel{\boldsymbol{\mu}}{(\boldsymbol{U})}=E(\boldsymbol{U})+q(\boldsymbol{U})$. In the case $\boldsymbol{U}^{0}=\overline{\boldsymbol{U}}+\boldsymbol{W}^{U}$ with small $W^{0}$, it is thus reasonable to consider the following approximation of the Kinetic Scheme

$$
\begin{aligned}
& \overline{\boldsymbol{U}}+\tilde{\boldsymbol{W}}(t, \boldsymbol{x})=\left\langle\stackrel{\rho}{\boldsymbol{\mu}}\left(\boldsymbol{U}^{0}(\boldsymbol{x}-t \boldsymbol{v}) ; \boldsymbol{v}\right), 1\right\rangle_{\boldsymbol{v}} \\
& \approx\left\langle\stackrel{\boldsymbol{\mu}}{ }(\overline{\boldsymbol{U}} ; \boldsymbol{v})+(E(\overline{\boldsymbol{U}} ; v)+q(\overline{\boldsymbol{U}} ; v)) \boldsymbol{W}^{0}(\boldsymbol{x}-t \boldsymbol{v}), 1\right\rangle_{\boldsymbol{v}} .
\end{aligned}
$$

According to our investigations in Section $6, E(\overrightarrow{\boldsymbol{U}} ; v) \boldsymbol{W}$ is the constraint function $\stackrel{\circ}{\mu}_{l i n}(\boldsymbol{W} ; \boldsymbol{v})$ for the linearized system (21). For the remainder term in (24) we introduce $\boldsymbol{\mu}_{q}(\boldsymbol{W} ; \boldsymbol{v})=q(\overline{\boldsymbol{U}} ; v) \boldsymbol{W}$ so that the linearized Kinetic Scheme is clearly seen to be the superposition of two linear Kinetic Schemes

$$
\tilde{\boldsymbol{W}}(t, x) \approx\left\langle\stackrel{\circ}{\mu}_{l i n}\left(\boldsymbol{W}^{0}(x-t v) ; \boldsymbol{v}\right), 1\right\rangle_{\boldsymbol{v}}+\left\langle\boldsymbol{\mu}_{q}\left(\boldsymbol{W}^{0}(\boldsymbol{x}-t \boldsymbol{v}) ; \boldsymbol{v}\right), 1\right\rangle_{\boldsymbol{v}}
$$


While the first contribution is the exact solution of the linear system (21) (and thus stable), the second one strongly depends on the selected curves and the interplay of these curves with the matrix $E$ which contains the information about the hyperbolic system. Stability problems of the scheme can originate only in the term related to the matrix $q$ defined in (23).

At this point it becomes obvious that the Kinetic Scheme is particularly well suited for those hyperbolic problems for which closed curve integrals over $E$ vanish. In this case, $q$ is identically zero and the linearized Kinetic Scheme is the optimal Kinetic Scheme for the linearized equation.

From the theory of differential one-forms it is known that vanishing closed curve integrals are related to exactness. Applied to $E=\mathcal{F}_{\xi}^{-1} \exp \left(-i \xi_{j} A^{j}\right)$, the condition reduces to the requirement that the mapping

$$
\boldsymbol{U} \mapsto \exp \left(-i \xi_{j} A^{j}(\boldsymbol{U})\right)=\sum_{n=0}^{\infty} \frac{(-i)^{n}}{n !}\left(\xi_{j} A^{j}(\boldsymbol{U})\right)^{n}
$$

must be exact (i.e. possess a primitive). It can easily be shown [13] that this is just another way of requiring the exactness of all products $\boldsymbol{U} \mapsto\left(\xi_{j} A^{j}(\boldsymbol{U})\right)^{n}$. Note that this observation is related to the results of Theorem 1. In particular, for all scalar conservation laws, the proposed constraint function yields a linearly stable scheme.

In case the exactness of all products $\left(\xi_{j} A^{j}\right)^{n}$ is not given, the additional term related to $q$ does not vanish and instabilities can occur if the Fourier transform of $q$ has amplifying modes.

\subsection{The modified equation approach}

In the general one-dimensional case, the Kinetic Scheme based on $\dot{\mu}$ yields a first order accurate solution to the problem

$$
\partial_{t} \boldsymbol{U}(t, x)+\partial_{x} \boldsymbol{F}(\boldsymbol{U}(t, x))=\mathbf{0}, \quad \boldsymbol{U}(0, x)=\boldsymbol{U}^{0}(x)
$$

(for $d=1$, the index in $\boldsymbol{F}^{1}$ and $A^{1}$ will be suppressed and $x$ and $v$ are scalars). However, by a simple Taylor expansion argument, one can check that the approximation obtained with the Kinetic Scheme is at least second order accurate to the so called modified equation

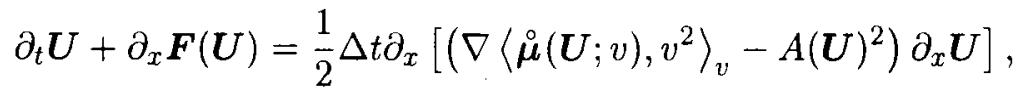

$$
\begin{aligned}
& \left.U\right|_{t=0}=U^{0}
\end{aligned}
$$

The modified equation (25) is a nonlinear advection diffusion equation with diffusion matrix $\frac{1}{2} \Delta t\left(\nabla\left\langle\dot{\boldsymbol{\mu}}, v^{2}\right\rangle_{v}-(A)^{2}\right)$. If this matrix has negative eigenvalues we expect 
(25) to behave like the ill posed backward heat equation which roughens the solution during the evolution. Since the Kinetic Scheme yields a good approximation to (25), a similar behavior is then expected for the Kinetic Scheme. This heuristic argument is the motivation for the following definition of stability.

Definition 2 Let $d=1$ and $\boldsymbol{F}=\boldsymbol{F}^{1}, A=A^{1}$. The kinetic scheme based on $\stackrel{\circ}{\mu}$ is called stable (in the sense of modified equation) if

$$
Q(\boldsymbol{U}):=\nabla\left\langle\stackrel{\boldsymbol{\mu}}{\boldsymbol{U}}(\boldsymbol{U} ; v), v^{2}\right\rangle_{v}-A(\boldsymbol{U})^{2}
$$

has only nonnegative eigenvalues for all $\boldsymbol{U} \in \mathcal{S}$.

We remark that, as in (16) or (17), one can show that $\left\langle E, v^{2}\right\rangle=(A)^{2}$. Using the relation $\nabla \stackrel{\boldsymbol{\mu}}{(\boldsymbol{U})}=E(\boldsymbol{U})+q(\boldsymbol{U})$, we thus conclude

$$
Q(\boldsymbol{U}) \boldsymbol{e}=\left\langle q(\boldsymbol{U} ; v) \boldsymbol{e}, v^{2}\right\rangle_{v}=\left.\frac{d}{d h} \oint_{C_{h}(\boldsymbol{U}, \boldsymbol{e})}(A)^{2}\right|_{h=0}
$$

In this formulation we see that $Q(\boldsymbol{U})$ can be interpreted as a measure of nonexactness of $(A)^{2}$ with respect to the family of curves $\left\{\Gamma_{\boldsymbol{U}}\right\}$.

\section{Scalar equations}

In the case of a single conservation law $(m=1)$, the fluxes $F^{j}$ are scalar functions defined on an interval $\mathcal{S} \subset \mathbb{R}$ and we can combine them in a vector

$$
\boldsymbol{F}(U)=\left(F^{1}(U), \ldots, F^{d}(U)\right)^{T}
$$

(note that $\boldsymbol{U}$ and the flux vectors $\boldsymbol{F}^{j}$ are replaced by non-bold symbols $U$ and $F^{j}$ since they are scalars in this section). Obviously, the $U$-derivative $\boldsymbol{F}^{\prime}$ of $\boldsymbol{F}$ is then given by $\boldsymbol{F}^{\prime}=\left(A^{1}, \ldots, A^{d}\right)$ and thus $\xi_{j} A^{j}=\boldsymbol{\xi} \cdot \boldsymbol{F}^{\prime}$. Since the inverse Fourier transform of $\exp \left(-i \boldsymbol{\xi} \cdot \boldsymbol{F}^{\prime}\right)$ is just the delta distribution shifted to $\boldsymbol{F}^{\prime}$, the constraint function (20) turns into

$$
\stackrel{\circ}{\mu}(U ; \boldsymbol{v})=\int_{0}^{U} \delta\left(\boldsymbol{v}-\boldsymbol{F}^{\prime}(s)\right) d s .
$$

We have already remarked in Section 8.1 that the Kinetic Scheme based on (26) is linearly stable. 


\subsection{Strictly convex flux in one dimension}

If we assume in addition that the space dimension is $d=1$ and that $F: \mathcal{S} \rightarrow \mathbb{R}$ is convex and twice continuously differentiable, we can easily transform (26) further. With the change of variables $\sigma=F^{\prime}(s)$ we get.

$$
\stackrel{\rho}{\mu}(U ; v)=\int_{F^{\prime}(0)}^{F^{\prime}(U)} \delta(v-\sigma) \frac{1}{F^{\prime \prime}(s)} d \sigma .
$$

Introducing $\mathcal{X}_{[a, b]}$ for the indicator function of the interval $[a, b]$ together with the convention that $\mathcal{X}_{[b, a]}=-\mathcal{X}_{[a, b]}$ for $a<b$, we conclude

$$
\stackrel{\circ}{\mu}_{\eta}(U ; v)=\frac{1}{F^{\prime \prime}(s)} \mathcal{X}_{\left[F^{\prime}(0), F^{\prime}(U)\right]}(v), \quad F^{\prime \prime}(s)=v .
$$

Using a different approach, the same constraint function has been derived in [2]. An extensive study of the resulting kinetic schemes can be found in [1]. It has been shown that the Kinetic Scheme based on $\stackrel{\circ}{\mu}$ is the exact solution of the Cauchy problem for $\partial_{t} U+\partial_{x} F(U)=0$, as long as no shocks develop. This is in accordance with the infinite order of consistency claimed in Theorem 1.

\subsection{The general case}

In general, the constraint function (26) cannot be simplified much further. However, it is possible to simplify the Kinetic Scheme based on $\stackrel{\leftrightarrow}{\mu}$. Setting

$$
\tilde{U}(t, \boldsymbol{x})=\left\langle\stackrel{\circ}{\mu}\left(U^{0}(\boldsymbol{x}-\boldsymbol{v} t) ; \boldsymbol{v}\right), 1\right\rangle_{\boldsymbol{v}}
$$

we obtain for any test function $\psi \in \mathscr{D}\left(\mathbb{R}^{d}\right)$

$$
\langle\tilde{U}(t, \boldsymbol{x}), \psi(\boldsymbol{x})\rangle_{\boldsymbol{x}}=\left\langle\stackrel{\leftrightarrow}{\mu}\left(U^{0}(\boldsymbol{x}) ; \boldsymbol{v}\right), \psi(\boldsymbol{x}+\boldsymbol{v} t)\right\rangle_{(\boldsymbol{x}, \boldsymbol{v})}
$$

and, using the structure of $\dot{\mu}$ in (26), we can evaluate the $v$-part of the dual pairing

$$
\begin{array}{r}
\langle\tilde{U}(t, \boldsymbol{x}), \psi(\boldsymbol{x})\rangle_{\boldsymbol{x}}=\left\langle\int_{0}^{U^{0}(\boldsymbol{x})} \psi\left(\boldsymbol{x}+F^{\prime}(s) t\right) d s, 1\right\rangle_{\boldsymbol{x}} \\
=\int_{\mathbb{R}}\left\langle\mathcal{X}_{\left[0, U^{0}(\boldsymbol{x})\right]}(s), \psi\left(\boldsymbol{x}+\boldsymbol{F}^{\prime}(s) t\right)\right\rangle_{\boldsymbol{x}} d s .
\end{array}
$$

Going over to the shifted $\boldsymbol{x}$ variable $\boldsymbol{x} \mapsto \boldsymbol{x}-\boldsymbol{F}^{\prime}(s) t$, we finally obtain

$$
\langle\tilde{U}(t, \boldsymbol{x}), \psi(\boldsymbol{x})\rangle_{\boldsymbol{x}}=\left\langle\int_{\mathbb{R}} \mathcal{X}_{\left[0, U^{0}\left(\boldsymbol{x}-\boldsymbol{F}^{\prime}(s) t\right)\right]}(s) d s, \psi(\boldsymbol{x})\right\rangle_{\boldsymbol{x}}
$$


In other words, the approximative solution $\tilde{U}$ of the entropy conservation law can be written as

$$
\tilde{U}(t, \boldsymbol{x})=\int_{\mathbb{R}} g(\boldsymbol{x}, s, t) d s
$$

where $g(\boldsymbol{x}, s, t)=\mathcal{X}_{\left[0, U^{0}\left(\boldsymbol{x}-\boldsymbol{F}^{\prime}(s) t\right)\right]}(s)$ solves the transport equation

$$
\partial_{t} g+A^{j}(s) \partial_{x_{j}} g=0, \quad g(\boldsymbol{x}, s, 0)=\mathcal{X}_{\left[0, U^{0}(\boldsymbol{x})\right]}(s)
$$

with $A^{j}=\left(F^{j}\right)^{\prime}$ and $s \in \mathbb{R}$. Note that (28) is not a free transport equation. Instead, the flow velocity of the kinetic particles is given by $\boldsymbol{F}^{\prime}(s)$. Compared to the original kinetic formulation (8) of the conservation law, the kinetic variable $s$ seems to be somewhat artificial since its dimension does not match the dimension of $\boldsymbol{x}$. In view of the above derivation, however, the relation to the standard kinetic formulation based on the free transport equation is clarified. In particular, $s$ is related to the state space integration occurring in the definition of the constraint function (26).

In [11] it is shown that a Kinetic Scheme based on (28) (and hence on (26)) converges to the unique entropy solution of the Cauchy problem $\partial_{t} U+\partial_{x_{j}} F^{j}(U)=0$. Also in [18] and [16] the relation between a transport equation of type (28) and the conservation law has been analyzed. In particular, it turns out that the kinetic approximation $\tilde{U}$ is the exact solution of the conservation law for small times, in accordance with Theorem 1.

\section{The one-dimensional Euler system}

In this example the state space $\mathcal{S}$ is three dimensional. The vector of unknowns consists of mass density $\rho$, momentum density $m$ and energy density $\rho \epsilon$. Important derived quantities are velocity $u=m / \rho$, temperature $T=(\gamma-1)\left(\epsilon-u^{2} / 2\right)$ and pressure $p=\rho T$ where $\gamma>1$ is a material constant. The state space $\mathcal{S}$ is a convex cone $\mathcal{S}=\left\{\rho(1, u, \epsilon)^{T} \mid \rho>0, T>0\right\}$. The nonlinear flux $\boldsymbol{F}$ is homogeneous of degree one so that its Jacobian $A$ is homogeneous of degree zero

$$
\boldsymbol{F}=\left(\begin{array}{c}
\rho u \\
\rho\left(u^{2}+T\right) \\
\rho(\epsilon+T) u
\end{array}\right), \quad A=\left(\begin{array}{ccc}
0 & 1 & 0 \\
\frac{1}{2}(\gamma-3) u^{2} & (3-\gamma) u & \gamma-1 \\
\frac{1}{2}(\gamma-2) u^{3}-\frac{\gamma}{\gamma-1} l^{\prime} u & \left(\frac{3}{2}-\gamma\right) u^{2}+\frac{\gamma}{\gamma-1} T^{\prime} & \gamma u
\end{array}\right)
$$

According to Theorem 1, the order of consistency of the Kinetic Scheme can only be higher than one if $A^{2}$ is exact. It turns out that the first row of $A^{2}$ is exact, the second row is exact only in the case $\gamma=3$ but then the third row is not exact so that the Kinetic Scheme based on $\stackrel{\rho}{\mu}$ is only first order accurate and depends on the selected curves $\Gamma_{U}$ in state space. A choice which is motivated by the structure of $\mathcal{S}$ and $\boldsymbol{F}$ are straight lines

$$
\Gamma_{\boldsymbol{U}}:=\{s \boldsymbol{U} \mid s \in(0,1]\} \quad \boldsymbol{U} \in \mathcal{S} .
$$


On these curves the Jacobian $A$ is constant due to homogeneity of $\boldsymbol{F}$ so that

$$
\stackrel{\circ}{\boldsymbol{\mu}}(\boldsymbol{U} ; v)=\int_{\Gamma_{\boldsymbol{U}}} \mathcal{F}_{\xi}^{-1} \exp (-i \xi A)=\mathcal{F}_{\xi}^{-1} \exp (-i \xi A(\boldsymbol{U})) \boldsymbol{U}
$$

To calculate $\mathcal{F}_{\xi}^{-1} \exp (-i \xi A)$ we diagonalize $A$ which has eigenvalues $\lambda_{1}=u, \lambda_{2}=$ $u-c$ and $\lambda_{3}=u+c$ with the sound speed $c=\sqrt{\gamma T}$. In a basis of right eigenvectors, the matrix $\exp (-i \xi A)$ has the form $\operatorname{diag}\left(\exp \left(-i \xi \lambda_{k}\right)\right)$ so that the inverse Fourier transform yields a linear superposition of $\delta\left(v-\lambda_{k}\right)$. Using the abbreviation

$$
f(\boldsymbol{U} ; v)=\rho\left(\frac{\gamma-1}{\gamma} \delta(v-u)+\frac{1}{2 \gamma} \delta(v-(u-c))+\frac{1}{2 \gamma} \delta(v-(u+c))\right)
$$

the resulting constraint function can be written as

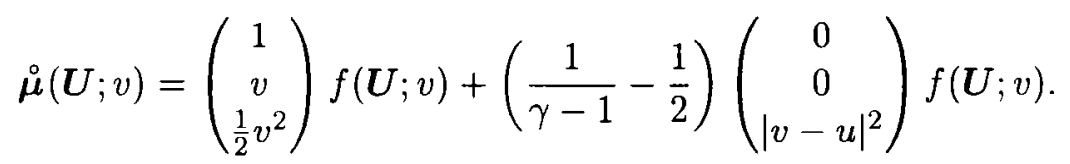

We remark that the same constraint function follows from the approach in [12]. We also note that, as in our introductory example (10), $\stackrel{\circ}{\mu}$ is actually based on a nonnegative, scalar function $f$. $\Lambda$ part from the case $\gamma=3$, howcver, it now consists of two contributions. Physically, the second term corresponds to the distribution of internal energy of the gas atoms. A similar splitting approach of the constraint function has been proposed in [17].

To analyze stability of the scheme we calculate the diffusion matrix $Q(\boldsymbol{U})$ given in Definition 2

$$
Q(\boldsymbol{U})=\left(\begin{array}{ccc}
0 & 0 & 0 \\
(\gamma-3) T u & (3-\gamma) T & 0 \\
\frac{\gamma^{2}-9 \gamma+6}{2(\gamma-1)} T u^{2}-\frac{\gamma}{\gamma-1} T^{2} & \frac{-2 \gamma^{2}+5 \gamma-3}{\gamma-1} T u & \gamma T
\end{array}\right)
$$

The cigenvalues are $0,(3-\gamma) T$ and $\gamma T$ so that the Kinetic Scheme is stable (in the sense of Definition 2) if $1<\gamma \leq 3$.

\section{A two-dimensional system}

As example, we consider the isentropic Euler system in two space dimensions. Under smooth conditions this system can be derived from (1) because the energy equation of the Euler system can be transformed into an equation for entropy which decouples from the system if the entropy is initially constant. The pressure $p$ which appears as source term in the momentum equation only depends on mass density and the constant value of entropy. To get hyperbolicity we restrict ourselves to pressure functions which satisfy $p^{\prime} \geq 0$. The vector of unknowns reduces to $U=\left(\rho, m_{1}, m_{2}\right)^{T}$ 
with $\mathcal{S}=\left\{\left(\rho, m_{1}, m_{2}\right)^{T} \mid \rho>0, m \in \mathbb{R}^{2}\right\}$ being a convex cone. Using again $\boldsymbol{u}=$ $m / \rho$, the fluxes are of the form

$$
\boldsymbol{F}^{1}=\left(\begin{array}{c}
\rho u_{1} \\
\rho u_{1}^{2}+p(\rho) \\
\rho u_{1} u_{2}
\end{array}\right), \quad \boldsymbol{F}^{2}=\left(\begin{array}{c}
\rho u_{2} \\
\rho u_{1} u_{2} \\
\rho u_{2}^{2}+p(\rho)
\end{array}\right)
$$

with Jacobians

$$
A^{1}=\left(\begin{array}{ccc}
0 & 1 & 0 \\
-u_{1}^{2}+p^{\prime}(\rho) & 2 u_{1} & 0 \\
-u_{1} u_{2} & u_{2} & u_{1}
\end{array}\right), \quad A^{2}=\left(\begin{array}{ccc}
0 & 0 & 1 \\
-u_{1} u_{2} & u_{2} & u_{1} \\
-u_{2}^{2}+p^{\prime}(\rho) & 0 & 2 u_{2}
\end{array}\right) \text {. }
$$

To see whether the Kinetic Schcmc bascd on $\stackrel{0}{\mu}$ can bc sccond order accurate we check the exactness of the products $A^{1} A^{1}, A^{2} A^{2}, A^{1} A^{2}$ and $A^{2} A^{1}$. In all cases, the first rows are exact but in the second and third rows we find nontrivial conditions

$$
\frac{p^{\prime}}{\rho}=\frac{1}{2} p^{\prime \prime}, \quad \frac{p^{\prime}}{\rho}=0, \quad p^{\prime \prime}=0 .
$$

These conditions are simultaneously only satisfied in the case of constant pressure and, as we shall see in Section 11.2, the Kinetic Scheme based on $\stackrel{\leftrightarrow}{\mu}$ then leads to infinite order of accuracy. For all other pressure laws, the Kinetic Scheme is always first order accurate.

\subsection{Non-constant pressure laws}

Choosing again the curves $\Gamma_{U}=\{s \boldsymbol{U} \mid s \in(0,1]\}$, we find after transformation to a basis of eigenvectors of $\xi_{j} A^{j}$ and integration

$$
\int_{\Gamma_{\boldsymbol{U}}} \exp \left(-i \xi_{j} A^{j}\right)=\rho\left(\begin{array}{c}
1 \\
i \partial_{\xi_{1}} \\
i \partial_{\xi_{2}}
\end{array}\right) e^{-i \boldsymbol{u} \cdot \boldsymbol{\xi}} \int_{0}^{1} \cos (c(s \rho)|\boldsymbol{\xi}|) d s
$$

Under the additional assumptions

$$
c(0)=0, \quad c^{\prime}>0, \quad \lim _{\sigma \rightarrow 0} \frac{\sigma}{c^{\prime}\left(c^{-1}(\sigma)\right)}=0 .
$$

which are satisfied for the practically relevant pressure laws $p(\rho)=C \rho^{\gamma}$ with $1<$ $\gamma \leq 3$ we can calculate the Fourier transform of (30) and get [13]

$$
\stackrel{\circ}{\boldsymbol{\mu}}(\boldsymbol{U} ; \boldsymbol{v})=\left(\begin{array}{c}
1 \\
v_{1} \\
v_{2}
\end{array}\right) a(\rho,|\boldsymbol{v}-\boldsymbol{u}|) \mathcal{X}_{[0, c(\rho))}(|\boldsymbol{v}-\boldsymbol{u}|) .
$$


We remark that the constraint function has again a structure similar to our initial example (10). In fact it consists of a scalar density

$$
f(\boldsymbol{U} ; \boldsymbol{v})=a(\rho,|\boldsymbol{v}-\boldsymbol{u}|) \mathcal{X}_{[0, c(\rho))}(|\boldsymbol{v}-\boldsymbol{u}|)
$$

which is multiplied by the vector $\left(\begin{array}{l}1 \\ v\end{array}\right)$. For pressure laws $p(\rho)=C \rho^{\gamma}$, the function $a$ is of the form

$$
a_{\gamma}(\rho,|v|)=\frac{\rho}{\pi(\gamma-1) c^{2}(\rho)}\left(\frac{1}{\sqrt{1-\nu^{2}}}-\frac{3-\gamma}{\gamma-1} \int_{\nu}^{1} \frac{\sigma^{\frac{4-2 \gamma}{\gamma-1}}}{\sqrt{\sigma^{2}-\nu^{2}}} d \sigma\right)
$$

with $\nu=|v| / c(\rho)$. Physically, $f$ in (31) is interpreted as particle distribution function so that a natural property would be $f \geq 0$. It turns out, however, that $a_{\gamma}$ and thus also $f$ is not always non-negative. For example, for $\gamma=7 / 5$ we have

$$
a_{7 / 5}(\rho,|v|)=\frac{5 \rho}{2 \pi c^{2}(\rho)} \frac{8 \nu^{4}-4 \nu^{2}-1}{\sqrt{1-\nu^{2}}}
$$

which changes sign at $\nu=\frac{1}{2} \sqrt{1+\sqrt{3}}$. To find a necessary condition for nonnegativity, we assume $f \geq 0$. Since $\stackrel{\boldsymbol{\mu}}{=}=\left(\begin{array}{l}1 \\ v\end{array}\right) f$ satisfies the consistency relations

$$
\langle\stackrel{\mu}{\boldsymbol{\mu}}(\boldsymbol{U} ; v), 1\rangle_{\boldsymbol{v}}=\boldsymbol{U}, \quad\left\langle\breve{\mu}(\boldsymbol{U} ; v), v_{j}\right\rangle_{\boldsymbol{v}}=\boldsymbol{F}^{j}(\boldsymbol{U})
$$

we get in particular

$$
\langle f(\boldsymbol{U} ; v), 1\rangle_{v}=\rho, \quad\left\langle f(\boldsymbol{U} ; v),|\boldsymbol{v}|^{2}\right\rangle_{\boldsymbol{v}}=\rho|\boldsymbol{u}|^{2}+2 p(\rho) .
$$

If $\boldsymbol{u}=\mathbf{0}, f$ is supported on $|v| \leq c(\rho)$ so that

$$
0<\left\langle f\left((\rho, \mathbf{0})^{T} ; \boldsymbol{v}\right), c(\rho)^{2}-|\boldsymbol{v}|^{2}\right\rangle_{v}=\rho c(\rho)^{2}-2 p(\rho) .
$$

Using $c^{2}=p^{\prime}$ we eventually find the condition $p^{\prime}(\rho)>2 p(\rho) / \rho$ for $f$ to be nonnegative. With the pressure laws $p(\rho)=C \rho^{\gamma}$ we get $\gamma>2$ so that the discovered non-definiteness in the case $\gamma=7 / 5$ is explained by the size of the support of $\stackrel{\mu}{\mu}$. We remark that in [15] a kinetic distribution of similar structure is proposed. In this case the scalar density is nonnegative and supported on $[0, \sqrt{2} c(\rho)]$ which is just large enough to exclude the above argument against positivity.

\subsection{The case of constant pressure}

In this particular case, the linear combination $\xi_{j} A^{j}$ can only be transformed into a Jordan matrix and $\hat{E}$ has the form

$$
\exp \left(-i \xi_{j} A^{j}\right)=e^{-i(u, \xi)}\left(\begin{array}{ccc}
1+i(\xi, u) & -i \xi_{1} & -i \xi_{2} \\
i(\xi, u) u_{1} & 1-i \xi_{1} u_{1} & -i \xi_{2} u_{1} \\
i(\xi, u) u_{2} & -i \xi_{1} u_{2} & 1-i \xi_{2} u_{2}
\end{array}\right)
$$


An easy but lengthy calculation shows that $\exp \left(-i \xi_{j} A^{j}\right)$ is exact for any $\boldsymbol{\xi} \in \mathbb{R}^{2}$. Consequently, $\stackrel{\leftrightarrow}{\mu}$ is independent of the chosen path and the resulting Kinetic Scheme is linearly stable. To carry out the integration, we choose $\Gamma_{\boldsymbol{U}}=\{s \boldsymbol{U} \mid s \in(0,1]\}$ since $\exp \left(-i \xi_{j} A^{j}\right)$ is constant along these paths. We find

$$
\int_{\Gamma_{\boldsymbol{U}}} \exp \left(-i \xi_{j} A^{j}\right)=\exp \left(-i \xi_{j} A^{j}(\boldsymbol{U})\right) \boldsymbol{U}=\rho\left(\begin{array}{l}
1 \\
\boldsymbol{u}
\end{array}\right) e^{-i \boldsymbol{u} \cdot \boldsymbol{\xi}}
$$

so that the Fourier transform is given by

$$
\stackrel{\mu}{\mu}(\boldsymbol{U} ; \boldsymbol{v})=\rho\left(\begin{array}{c}
1 \\
v_{1} \\
v_{2}
\end{array}\right) \delta(\boldsymbol{v}-u) .
$$

In [13] it is shown that for smooth initial values and small times the Kinetic Scheme based on (32) yields the exact solution of the problem which is in accordance with the infinite order of consistency.

\section{Isentropic Euler equations in one dimension}

Similar to the case of two-dimensional isentropic Euler equations we now have $\boldsymbol{U}=$ $(\rho, m)^{T}$ with $\mathcal{S}=\left\{(\rho, m)^{T} \mid \rho>0, m \in \mathbb{R}\right\}$ and

$$
\boldsymbol{F}=\left(\begin{array}{c}
\rho u \\
\rho u^{2}+p(\rho)
\end{array}\right), \quad A=\left(\begin{array}{cc}
0 & 1 \\
-u^{2}+p^{\prime} & 2 u
\end{array}\right), \quad u=\frac{m}{\rho} .
$$

Again, the case of constant pressure leads to $\stackrel{\circ}{\mu}=\rho\left(\begin{array}{l}1 \\ v\end{array}\right) \delta(v-u)$. This constraint function has been used by other authors to show the relation between the constant pressure system and kinetic theory (see [4] and the references therein). We will not repeat our argument from the previous section and restrict ourselves to the case $c=\sqrt{p^{\prime}}>0$. Moreover, we assume $p^{\prime \prime}>0$. The exponential $\hat{E}$ is given by

$$
\exp (-i \xi A)=\left(\begin{array}{c}
1 \\
i \partial_{\xi}
\end{array}\right) \frac{e^{-i \xi u}}{c}(c \cos (c \xi)+i u \sin (c \xi)-i \sin (c \xi))
$$

and is exact if the condition $p(\rho) / \rho=p^{\prime \prime}(\rho) / 2$ is satisfied which singles out the pressure law $p(\rho)=C \rho^{3}$. (For this special relation it is known that the isentropic system decouples into two independent Burgers equations.) Since the exactness of $\exp (-i \xi A)$ implies the exactness of all powers $A^{n}$, Theorem 1 implies that $\stackrel{\rho}{\mu}$ leads to a Kinetic Scheme of infinite order. For all other pressure laws, not even $A^{2}$ is exact so that the Kinetic Scheme is always first order accurate and the distribution $\stackrel{\mu}{\mu}$ depends on the selectcd curvcs. In this cxample we will investigate different families of curves. Of course,

$$
\Gamma_{\boldsymbol{U}}=\{s \boldsymbol{U} \mid s \in(0,1]\}, \quad \boldsymbol{U} \in \mathcal{S}
$$


is again a reasonable choice. However, there are other curves which are strongly connected to the structure of $\boldsymbol{F}$. They are given as integral curves of the right eigenvectors $\boldsymbol{r}_{1}=\frac{\rho}{2 c}\left(\begin{array}{c}1 \\ u-c\end{array}\right)$ and $\boldsymbol{r}_{2}=\frac{\rho}{2 c}\left(\begin{array}{c}1 \\ u+c\end{array}\right)$ of $A$, or equivalently as coordinate lines of the system of Riemann invariants [21]

$$
\left(\begin{array}{l}
W_{1} \\
W_{2}
\end{array}\right)=\left(\begin{array}{l}
\tilde{c}(\rho)-u \\
\tilde{c}(\rho)+u
\end{array}\right), \quad \tilde{c}(\rho)=\int_{0}^{\rho} \frac{c(\sigma)}{\sigma} d \sigma .
$$

For the calculation of $\stackrel{\AA}{\boldsymbol{\mu}}$ along $\boldsymbol{r}_{1}$-curves we first need a parameterization $\boldsymbol{\gamma}(\boldsymbol{U}, s)$. By definition, $\boldsymbol{U} \in \mathcal{S}$ is located on the $\boldsymbol{r}_{1}$-curve corresponding to $W_{2}=W_{2}(\boldsymbol{U})$. A simple parameterization is therefore given by

$$
\gamma(\boldsymbol{U}, s):=\left(\begin{array}{c}
s \rho \\
s \rho\left(W_{2}(\boldsymbol{U})-\tilde{c}(s \rho)\right)
\end{array}\right) \quad s \in(0,1] .
$$

Completing the calculation, we obtain

$$
\stackrel{\circ}{\boldsymbol{\mu}}(\boldsymbol{U} ; v)=\left(\begin{array}{l}
1 \\
v
\end{array}\right)\left[(\tilde{c}+c)^{-1}\right]^{\prime}(u-v+\tilde{c}(\rho)) \mathcal{X}_{[-c(\rho), \tilde{c}(\rho)]}(v-u) .
$$

Note that the $v$-support of $\stackrel{\circ}{\mu}$ is in general not symmetric with respect to $u$. Only in the case $c=\tilde{c}$, which is equivalent to $p=C \rho^{3}$, we get symmetry.

Using the $\boldsymbol{r}_{2}$-curves we obtain in a completely analogous manner

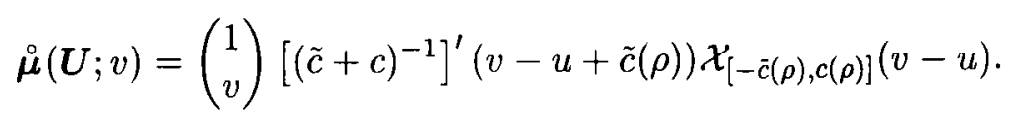

The support is again unsymmetric but the role of $\tilde{c}$ and $c$ has exchanged.

A symmetric distribution is obtained with the curves (34) which are in some sense a compromise between $\boldsymbol{r}_{1}$ and $\boldsymbol{r}_{2}$-curves. Indeed, the tangent vector $\rho\left(\begin{array}{l}1 \\ u\end{array}\right)$ is, up to the factor $1 / 2 c$, just the average $\frac{1}{2}\left(\boldsymbol{r}_{1}+\boldsymbol{r}_{2}\right)$. We obtain

$$
\stackrel{\circ}{\mu}(\boldsymbol{U} ; v)=\left(\begin{array}{l}
1 \\
v
\end{array}\right)\left[c^{-1}\right]^{\prime}(|v-u|) \mathcal{X}_{[0, c(\rho)]}(|v-u|) .
$$

This constraint function has been derived in [15] with a different approach.

We remark that in the particular case $\gamma=3$, all the constraint functions coincide because the chosen path of integration has no influence. Also, $c(\rho)=\tilde{c}(\rho)=\sqrt{3 C} \rho$ so that $\left[c^{-1}\right]^{\prime}$ is a constant and $\stackrel{\circ}{\mu}$ is determined by the scalar constraint function

$$
f(\boldsymbol{U} ; v)=\frac{1}{\sqrt{3 C}} \mathcal{X}_{[0, c(\rho)]}(|v-u|) .
$$

Recently, Brenier and Corrias [3] have derived a hierarchy of hyperbolic systems which includes the isentropic Euler system with $\gamma=3$ as a special case. Other systems in the hierarchy involve a higher dimensional state space $\mathcal{S}$. By construction, 
smooth solutions of these systems can be written in the form of the Kinetic Scheme with a particular constraint function. Since exactness of the solution implies infinite order of consistency of the Kinetic Scheme, we conclude with Theorem 1, that the constraint functions used in [3] essentially coincide with those introduced here.

To complete the example, we take a look at stability. For each of the chosen families of curves one can show that the diffusion matrix has the form

$$
Q(\boldsymbol{U})=\left(3 \frac{p}{\rho}-p^{\prime}\right)\left(\begin{array}{ll}
0 & 0 \\
* & 1
\end{array}\right)
$$

where the entry $*$ is equal to $-u$ for the straight lines (31), equal to $c-u$ for the $\boldsymbol{r}_{1}$-curves and $-(u+c)$ for the $\boldsymbol{r}_{2}$-curves. Consequently, the Kinetic Schemes are stable if and only if $3 p / \rho-p^{\prime} \geq 0$. In the case $p=C \rho^{\gamma}$ this is equivalent to $\gamma \leq 3$.

\section{The p-system}

The final example concerns the nonlinear wave equation

$$
\partial_{t}^{2} \varphi+\partial_{x}^{2} p(\varphi)=0 \quad p^{\prime}<0, p^{\prime \prime}>0 .
$$

Motivated by the linear case $p(\varphi)=-c^{2} \varphi$ we introduce the wave speed $c(\varphi)=$ $\sqrt{-p^{\prime}(\varphi)}$. As important example, we mention the isentropic Euler equation in Lagrangian coordinates. Here $\varphi$ is interpreted as specific volume $1 / \rho$ and $p$ is the pressure which typically decreases with decreasing $\rho$. To treat equation (35) in our context we first transform it into a system of first order equations, the so called psystem [21]. Setting $U_{1}=\varphi$ which ranges in some interval $I \subset \mathbb{R}$ and $U_{2}=\partial_{t} \varphi \in \mathbb{R}$, we obtain a system with flux vector

$$
\boldsymbol{F}=\left(\begin{array}{c}
-U_{2} \\
p\left(U_{1}\right)
\end{array}\right), \quad \boldsymbol{U} \in \mathcal{S}=I \times \mathbb{R}
$$

After diagonalizing the Jacobian $A$, we find

$$
\exp (-i \xi A)=\left(\begin{array}{c}
1 \\
-i \partial_{\xi}
\end{array}\right)\left(\cos (\xi c) \quad \frac{1}{c} i \sin (\xi c)\right)
$$

which is only exact in the linear casc where $c$ is independent of $U_{1}$. Conscquently, properties of $\boldsymbol{\mu}$ are influenced by the chosen curves $\Gamma_{\boldsymbol{U}}$.

In general, the state space $\mathcal{S}$ will not possess a distinguished point like the origin in the previous examples. We thus pick any $\tilde{U}_{1} \in I$ and use $\tilde{\boldsymbol{U}}=\left(\begin{array}{c}\tilde{U}_{1} \\ 0\end{array}\right)$ as starting point for the family of curves which we again take as integral lines of the right eigenvectors or, equivalently, as piecewise coordinate lines of a system of Riemann 
invariants (see Fig. 2). As mapping $\boldsymbol{H}$ from the Riemann invariants $\boldsymbol{W}$ to the conservative variables $\boldsymbol{U}$ we choose

$$
\left(\begin{array}{l}
W_{1} \\
W_{2}
\end{array}\right)=\left(\begin{array}{l}
U_{2}+\bar{c}\left(U_{1}\right) \\
U_{2}-\bar{c}\left(U_{1}\right)
\end{array}\right), \quad \bar{c}=\int_{\tilde{U}_{1}}^{U_{1}} c(\sigma) d \sigma .
$$

such that the image of the reference state $\tilde{U}$ is just $\tilde{\boldsymbol{W}}=\mathbf{0}$.
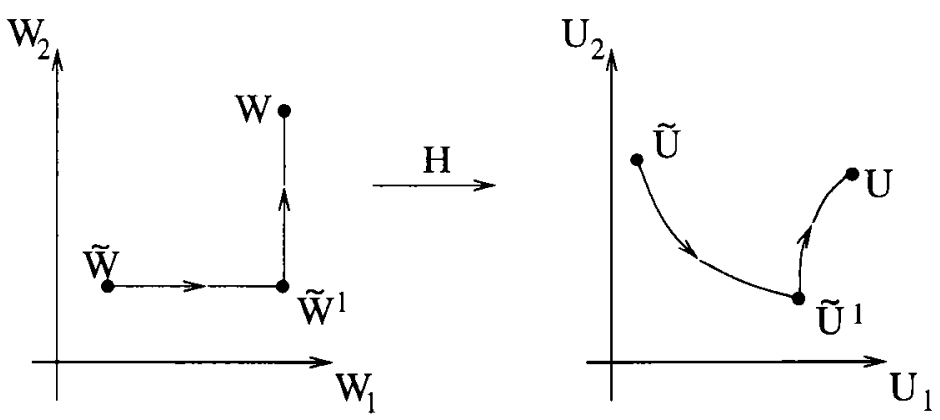

Figure 2: Curve along characteristic fields

The point where the curve switches from the first to the second field is denoted $\tilde{W}^{1}$ respectively $\tilde{\boldsymbol{U}}^{1}=\boldsymbol{H}\left(\tilde{\boldsymbol{W}}^{1}\right)$. Calculating the curve integral over $E=\mathcal{F}_{\xi}^{-1} \exp (-i \xi A)$, we eventually find

$$
\stackrel{\circ}{\boldsymbol{\mu}}(\boldsymbol{U} ; v)=\left[c^{-1}\right]^{\prime}(|v|)\left(\begin{array}{c}
1 \\
-v
\end{array}\right)\left(\mathcal{X}_{\left[-c\left(\tilde{U}_{1}^{1}\right), c\left(\tilde{U}^{1}\right)\right]}(v)+\mathcal{X}_{\left[c\left(\tilde{U}_{1}^{1}\right), c\left(U_{1}\right)\right]}(v)\right) .
$$

Note that the arbitrary point $\tilde{\boldsymbol{U}}$ decisively determines the support of $\hat{\mu}$. In fact, this arbitrariness can be the reason for instability. To see this, we calculate the diffusion matrix

$$
Q(\boldsymbol{U})=\left(c\left(\tilde{U}_{1}^{1}\right)-c\left(U_{1}\right)\right)\left(\begin{array}{cc}
0 & 0 \\
c\left(U_{1}\right) & 1
\end{array}\right)
$$

which has a negative eigenvalue if $U_{1}<\tilde{U}_{1}^{1}$ (note that $c$ is decreasing due to $p^{\prime \prime}>0$ ). Consequently, the state space splits into a stable and an unstable region which are separated by the $r_{2}$-curve through $\tilde{U}$.

\section{Conclusion}

We have presented a general construction principle for constraint functions used in Kinetic Schemes which makes the approach applicable to general hyperbolic systems. The principle extends and generalizes several concepts proposed by other authors. Moreover, a specific criterion is presented which singles out a certain class of equations for which the kinetic approach is particularly well suited. This class includes 
all linear hyperbolic systems, non-linear scalar equations as well as some special non-linear systems. It remains an open problem, whether the kinetic approach is as helpful in studying these systems as it has been in the case of scalar equations.

Although the construction of the constraint functions is motivated by linear theory and consistency analysis, the resulting schemes can of course be used for the approximation of weak solutions. In fact, whenever the approach reduces to known concepts, a corresponding analysis is already available.

\section{A Appendix}

The aim of the appendix is to describe the mathematical structure of the constraint function $\stackrel{\circ}{\mu}$. At the same time, we are going to extend the concept of kinetic formulations to entropy conservation laws related to the system (7). Here, a convex scalar function $\eta: \mathcal{S} \mapsto \mathbb{R}$ is an entropy function with entropy fluxes $\varphi^{j}: \mathcal{S} \mapsto \mathbb{R}$ provided

$$
\nabla^{T} \eta \nabla \boldsymbol{F}^{j}=\nabla^{T} \varphi^{j}, \quad j=1, \ldots, d
$$

where $\nabla^{T} \eta=(\nabla \eta)^{T}$. Of course, differentiability of $\eta$ and $\varphi^{j}$ is required. We will also assume that $\eta(\mathbf{0})=0$ as well as $\varphi^{j}(\mathbf{0})=0$ which can be achieved by subtracting the value at zero. If $\boldsymbol{U}$ is a smooth solution of (7), relation (36) implies that $\eta(\boldsymbol{U})$ satisfies an additional conservation law

$$
\partial_{t} \eta(\boldsymbol{U})+\partial_{x_{j}} \varphi^{j}(\boldsymbol{U})=0
$$

A kinetic formulation of the conservation law (37) is obtained with an entropy constraint function $\mu_{\eta}(\boldsymbol{U} ; \boldsymbol{v})$ which satisfies the consistency condition

$$
\left\langle\mu_{\eta}(\boldsymbol{U}), 1\right\rangle_{v}=\eta(\boldsymbol{U}), \quad\left\langle\mu_{\eta}(\boldsymbol{U}), v_{j}\right\rangle_{\boldsymbol{v}}=\varphi^{j}(\boldsymbol{U})
$$

Then (37) is formally equivalent to the constrained evolution

$$
\partial_{t} \Phi+v_{j} \partial_{x_{j}} \Phi=0, \quad \Phi(t, \boldsymbol{x}, \boldsymbol{v})=\mu_{\eta}(\boldsymbol{U}(t, \boldsymbol{x}) ; \boldsymbol{v}) .
$$

Note that our original considerations are included in this approach by choosing special linear entropy-entropyflux pairs

$$
\eta(\boldsymbol{U})=U_{i}, \quad \varphi^{j}(\boldsymbol{U})=F_{i}^{j}(\boldsymbol{U}), \quad i \in\{1, \ldots, d\} .
$$

With this choice, which clearly satisfies relation (36), the conservation law (37) is just the $i^{\text {th }}$ member of the system of conservation laws (7). This observation enables us to investigate the constraint functions for the system (7) and the entropy constraint functions for (37) simultaneously. 
As entropy constraint function, we propose

$$
\stackrel{\circ}{\mu}_{\eta}(\boldsymbol{U})=\int_{\Gamma_{\boldsymbol{U}}} \nabla^{T} \eta E, \quad E(\boldsymbol{U})=\mathcal{F}_{\boldsymbol{\xi}}^{-1} \exp \left(-i \xi_{j} A^{j}(\boldsymbol{U})\right)
$$

which reduces to (20) for the entropies (39) and satisfies (38). Indeed, using the fact that $\langle E, 1\rangle=\mathrm{I}$ and $\left\langle E, v_{k}\right\rangle=A^{k}(\boldsymbol{U})$ for every $\boldsymbol{U} \in \mathcal{S}$, we get

$$
\left\langle\stackrel{\circ}{\eta}_{\eta}(\boldsymbol{U}), 1\right\rangle_{\boldsymbol{v}}=\int_{\Gamma_{\boldsymbol{U}}} \nabla^{T} \eta\langle E, 1\rangle=\int_{\Gamma_{\boldsymbol{U}}} \nabla^{T} \eta=\eta(\boldsymbol{U})
$$

and

$$
\left\langle\stackrel{\circ}{\mu}_{\eta}(\boldsymbol{U}), v_{k}\right\rangle_{v}=\int_{\Gamma_{U}} \nabla^{T} \eta\left\langle E, v_{k}\right\rangle=\int_{\Gamma_{U}} \nabla^{T} \eta A^{k}=\int_{\Gamma_{U}} \nabla \varphi^{k}=\varphi^{k}(\boldsymbol{U})
$$

To describe the mathematical structure of $(40)$, we begin with some remarks on the function

$$
\hat{E}(\boldsymbol{U} ; \boldsymbol{\xi})=\exp \left(-i \xi_{j} A^{j}(\boldsymbol{U})\right), \quad A^{j}=\nabla \boldsymbol{F}^{j}
$$

(for details and proofs we refer to [13]). At the core of the investigations is a result from the theory of linear hyperbolic systems which is based on the following Lemma [23].

Lemma 3 Let $M$ be any $m \times m$ matrix. There is a constant $C_{m}$ depending only on $m$ such that

$$
\|\exp (i M)\| \leq C_{m}(1+\|M\|)^{m} \exp (I(M))
$$

where $I(M)$ is the largest absolute value of the imaginary parts of the eigenvalues of $M$.

If we take in particular $M=\xi_{j} A^{j}(\boldsymbol{U})$, hyperbolicity implies that all eigenvalues of $M$ are real so that $I(M)=0$. This leads to the estimate

$$
\|\hat{E}(\boldsymbol{U} ; \boldsymbol{\xi})\| \leq C_{\boldsymbol{U}}(1+|\boldsymbol{\xi}|)^{m}
$$

where $C_{\boldsymbol{U}}$ depends on $\max _{j=1}^{m}\left\|A^{j}(\boldsymbol{U})\right\|$. In particular, each component of the matrix $\hat{E}(\boldsymbol{U} ; \boldsymbol{\xi})$ grows at most polynomially in $|\boldsymbol{\xi}|$. Thus, $\hat{E}(\boldsymbol{U})$ can be interpreted as a matrix of regular, tempered distributions on $\mathbb{R}^{d}$, i.e. $\hat{E}(\boldsymbol{U}) \in \mathscr{S}^{\prime}\left(\mathbb{R}^{d}\right)^{m \times m}$. Moreover, $U \mapsto \hat{E}(U)$ is continuous as a mapping from $\mathcal{S}$ to $\left[\mathscr{S}^{\prime}\right]^{m \times m}$. Since the $\boldsymbol{\xi}$ dependence of $\hat{E}$ is analytic, the Paley-Wiener theorem implies that for each $U \in \mathcal{S}$ the inverse Fourier transform $E(U)=\mathcal{F}^{-1} \hat{E}(U)$ is a matrix of compactly supported distributions. The mapping $U \mapsto E(U)$ from $\mathcal{S}$ to $\left[\mathscr{E}^{\prime}\right]^{m \times m}$ is also continuous.

Our basic assumptions on the curves $\Gamma_{U} \subset \mathcal{S}$ which appear in the definition of the constraint functions (40) are listed in the following definition. The requirements are satisfied, for example, by curves which consist of piecewise constant segments along the coordinate axes or smooth transformations thereof. 
Definition 4 (admissible family of curves)

Let $\mathcal{S} \subset \mathbb{R}^{m}$ be open with $\mathbf{0} \in \overline{\mathcal{S}}$. A function $\gamma: \mathcal{S} \times[0,1] \mapsto \overline{\mathcal{S}}$ is called admissible family of curves in $\mathcal{S}$ if $\boldsymbol{\gamma}(\boldsymbol{U}, 0)=\mathbf{0}$, and $\boldsymbol{\gamma}(\boldsymbol{U}, 1)=\boldsymbol{U}$, if $\boldsymbol{\gamma}: \mathcal{S} \times[0,1] \mapsto \overline{\mathcal{S}}$ is continuous, and $\gamma(\boldsymbol{U}, \cdot):[0,1] \mapsto \overline{\mathcal{S}}$ is piecewise $C^{1}$, if $\dot{\boldsymbol{\gamma}} \in \mathbb{L}_{\text {loc }}^{\infty}\left(\mathcal{S} \times[0,1], \mathbb{R}^{m}\right)$, and $\nabla \dot{\gamma} \in \mathbb{L}_{\text {loc }}^{\infty}\left(\mathcal{S} \times[0,1], \mathbb{R}^{m}\right)$, where $\nabla$ refers to the $\boldsymbol{U}$-derivative and the dot to the $s$-derivative. The image of $\gamma(U, \cdot)$ is denoted $\Gamma_{U}$.

Since our main interest is to integrate $\nabla^{T} \eta E$ along the curves $\Gamma_{\boldsymbol{U}}$, we introduce suitable families of curves for this purpose.

Definition 5 (F-admissible family)

Let $\boldsymbol{\gamma}$ be an admissible family in $\mathcal{S}$. A measurable function $f: \mathcal{S} \mapsto \mathbb{R}^{m^{\prime}}$ is called locally bounded on $\gamma$ if for any compact set $K \subset \mathcal{S}$

$$
\|f(\gamma)\|_{\mathbb{L}^{\infty}(K \times[0,1])}<\infty .
$$

The family $\boldsymbol{\gamma}$ is called $\boldsymbol{F}$-admissible if the derivatives $A^{j}=\nabla \boldsymbol{F}^{j}$ are locally bounded on $\gamma$.

We remark that Definition 5 is only necessary if we want to include cases where the curves $\Gamma_{\boldsymbol{U}}$ touch $\partial \mathcal{S}$. Otherwise, the $\boldsymbol{F}$-admissibility is an immediate consequence of continuity of the matrices $A^{j}$.

In the following, we always assume that $\eta$ is an entropy which is locally bounded on the $\boldsymbol{F}$-admissible family $\boldsymbol{\gamma}$. Then, the linear mapping

$$
\phi \mapsto \int_{0}^{1}\left\langle\nabla^{T} \eta(\boldsymbol{\gamma}(\boldsymbol{U}, s)) E(\boldsymbol{\gamma}(\boldsymbol{U}, s) ; \boldsymbol{v}) \dot{\boldsymbol{\gamma}}(\boldsymbol{U}, s), \phi(\boldsymbol{v})\right\rangle_{\boldsymbol{v}} d s, \quad \phi \in \mathscr{E}\left(\mathbb{R}^{d}\right)
$$

defines a compactly supported distribution $\stackrel{\circ}{\eta}_{\eta}(U) \in \mathscr{E}^{\prime}\left(\mathbb{R}^{d}\right)$ which depends continuously on $\boldsymbol{U}$. This result, which is proved in [13], justifies the notation (40). A careful analysis of the continuity properties of the mapping $\boldsymbol{U} \mapsto \stackrel{\circ}{\mu} \eta(\boldsymbol{U}) \in \mathscr{E}^{\prime}\left(\mathbb{R}^{d}\right)$ motivates the following definition of a larger set of constraint functions which contains $\ddot{\mu}_{\eta}$ as a special case.

Definition $6 \quad$ By $\mathscr{K}$ we denote the set of all continuous $\mathscr{E}^{\prime}\left(\mathbb{R}^{d}\right)$ valued functions $\mu$ on $\mathcal{S}$ which satisfy for any compact $K \subset \mathcal{S}$ and any $\phi \in \mathscr{E}\left(\mathbb{R}^{d}\right)$

$$
\left|\langle\mu(\boldsymbol{U} ; \boldsymbol{v}), \phi(\boldsymbol{v})\rangle_{\boldsymbol{v}}\right| \leq C_{K} \sum_{|\boldsymbol{\alpha}| \leq N} \sup _{\boldsymbol{v} \mid \leq r_{K}}\left|D^{\boldsymbol{\alpha}} \phi(\boldsymbol{v})\right| \quad \forall \boldsymbol{U} \in K
$$

where $N \in \mathbb{N}$ depends only on $\mu$ and $C_{K}, r_{K}>0$ are constants depending on $\mu$ and $K$. 
For $\mu \in \mathscr{K}$ the operations which typically appear in the framework of Kinetic Schemes are justified. Under certain conditions on $U^{0}$, for example, the expression

$$
\eta_{\mu}(t, \boldsymbol{x}):=\left\langle\mu\left(\boldsymbol{U}^{0}(\boldsymbol{x}-\boldsymbol{v} t) ; \boldsymbol{v}\right), 1\right\rangle_{\boldsymbol{v}}
$$

defines a $C^{\infty}$-smooth function $t \mapsto \eta_{\mu}(t, \cdot)$ which maps $\mathbb{R}$ into the set of generalized functions $\mathscr{D}^{\prime}\left(\mathbb{R}^{d}\right)$. More precisely, we assume that the range of the measurable initial value $\boldsymbol{U}^{0}$ is a compact subset of $\mathcal{S}$, i.e $U^{0}\left(\mathbb{R}^{d}\right) \subset \subset \mathcal{S}$. This implies that $\boldsymbol{U}^{0}$ is bounded and stays away from $\partial \mathcal{S}$. Then, for a given $\phi \in \mathscr{E}\left(\mathbb{R}^{d}\right)$ and $\mu \in \mathscr{K}$,

$$
\psi \mapsto\left\langle\mu\left(\boldsymbol{U}^{0}(\boldsymbol{x}-\boldsymbol{v} t) ; \boldsymbol{v}\right), \psi(\boldsymbol{x}) \phi(\boldsymbol{v})\right\rangle_{(\boldsymbol{x}, \boldsymbol{v})} \quad \psi \in \mathscr{D}\left(\mathbb{R}^{d}\right)
$$

defines a distribution in $\boldsymbol{x}$ which we denote $\left\langle\mu\left(\boldsymbol{U}^{0}(\boldsymbol{x}-\boldsymbol{v} t) ; \boldsymbol{v}\right), \phi(\boldsymbol{v})\right\rangle_{\boldsymbol{v}}$. Choosing $\phi \equiv 1$, we see that for each $t \in \mathbb{R}$ the approximation (41) is an element of $\mathscr{D}^{\prime}\left(\mathbb{R}^{d}\right)$ and the dependence on $t$ is $C^{\infty}$.

\section{References}

[1] M. B̈̈CKER, Kinetische Verfahren für nichtlineare skalare Erhaltungsgleichungen, Ph.D. thesis, Universität Kaiserslautern, Arbeitsgruppe Technomathematik, 1993

[2] M. B̈̈CKER, K. DRESSLER, A kinetic method for strictly nonlinear scalar conservation laws, ZAMP, Vol.42, 1991

[3] Y. Brenier, L. Corrias, A kinetic formulation for multi-branch entropy solutions of scalar conservation laws, Ann. Inst. Henri Poincare, Anal. Non Lincairc 15, No.2, 169 190, 1998

[4] Y. BRENIER, E. GRENIER, Sticky particles and scalar conservation laus, SIAM J. Numer. Anal., 35, 2317-2328, 1997

[5] C. Cercignani, The Boltzmann Equation And Its Applications, Springer, 1988

[6] S. M. DeshPande, Kinetic theory based new upwind methods for inviscid compressible flows, AIAA paper 86-0275, American Institute of Aeronautics and Astronautics, New York, 1986

[7] S. M. Deshrande, J. C. Mandal, Kinetic flux-vector splitting (KFVS) for the Euler equation, Report 87 FM 2, Dept. Aerospace Eng., I.I.Sc., Bangalore, 1987

[8] Sanjay S. Deshrande, A Boltzmann-Taylor-Galerkin FEM for compressible Euler equations, in Proceedings of the 14th international conference on numerical methods in fluid dynamics, Bangalore, India, 11-15 July, 1994, Deshpande, S. M. (ed.) et al., Springer-Verlag. Lect. Notes Phys. 453, 91-95, 1995 
[9] S. M. Deshrande and O. Pironneau, A kinetic Fourier scheme, C. R. Acad. Sci., Paris, Ser. I 321, No.8, 1011-1016, 1995

[10] J. L. Estivalezes, P. VilledieU, High-order positivity preserving kinelic schemes for the compressible Euler equations, SIAM J. Numer. Anal.,33,20502067,1996

[11] Y. Giga, T. Mryakawa, A kinctic construction of global solutions of first order quasilinear equations, Duke Math. J., 50, 505-515, 1983

[12] A. Harten, P. D. LAX, B. van Leer, On upstream differencing and Godunov-type schemes for hyperbolic conservation laws, SIAM Rev., 25,35-61, 1983

[13] M. Junk, Kinetic Schemes: A new approach and applications, Ph.D. thesis, Universität Kaiserslautern, Shaker Verlag, 1997

[14] M. Junk, Exponentially exact conservation laws, in preparation

[15] S. Kaniel, A Kinetic Model for the Compressible Flow Equation, Indiana Univ. Math. J., Vol.37, No.3, 1988

[16] P. L. Lions, B. Perthame, E. Tadmor, A kinetic formulation of multidimensional scalar conservation laws and related equations, J. Amer. Math. Soc., 7, 169-191, 1994

[17] B. PeRT'HAme, Boltzmann type schemes for gas dynamics and the entropy property, SIAM J. Numer. Anal. 27, No.6, 1405-1421, 1990

[18] B. Perthame, E. Tadmor, A kinetic equation with kinetic entropy functions for scalar conservation laws, Comm. Math. Phys., 136, 501-517, 1991

[19] D. I. Pullin, Direct simulation methods for compressible inviscid ideal-gas flow, J. Comput. Phys., 34, 231-244, 1980

[20] R. D. REITz, One-dimensional compressible gas dynamic calculations using the Boltzmann equation, J. Comput. Phys., 42, 108-123, 1981

[21] J. SMOLLER, Shock Waves and Reaction-Diffusion Equations, Springer, 1983

[22] R. H. Sanders, K. H. Prendergast, On the origin of the 3 kiloparsec arm, Astrophys. J., 188, 489-500, 1974

[23] F. Treves, Basic Linear Partial Differential Equations, Academic Press, 1975 